\title{
Review
}

\section{The proteasome: Overview of structure and functions}

\author{
By Keiji TANAKA*1,† \\ (Communicated by Takao SEKIYA, M.J.A.)
}

\begin{abstract}
The proteasome is a highly sophisticated protease complex designed to carry out selective, efficient and processive hydrolysis of client proteins. It is known to collaborate with ubiquitin, which polymerizes to form a marker for regulated proteolysis in eukaryotic cells. The highly organized proteasome plays a prominent role in the control of a diverse array of basic cellular activities by rapidly and unidirectionally catalyzing biological reactions. Studies of the proteasome during the past quarter of a century have provided profound insights into its structure and functions, which has appreciably contributed to our understanding of cellular life. Many questions, however, remain to be elucidated.
\end{abstract}

Keywords: proteasome, ubiquitin, intracellular proteolysis, multisubunit complex, molecular chaperone

\section{Introduction}

The proteasome is a large protein complex responsible for degradation of intracellular proteins, a process that requires metabolic energy. Polymerization of ubiquitin, a key molecule known to work in concert with the proteasome, serves as a degradation signal for numerous target proteins; the destruction of a protein is initiated by covalent attachment of a chain consisting of several copies of ubiquitin (more than four ubiquitin molecules), through the concerted actions of a network of proteins, including the E1 (ubiquitin-activating), E2 (ubiquitin-conjugating) and E3 (ubiquitin-ligating) enzymes. ${ }^{1), 2)}$ The polymerized ubiquitin chain acts as a signal that shuttles the target proteins to the proteasome, where the substrate is proteolytically broken down. For accurate selection of the proteins, numerous enzymes (e.g., 2 E1 proteins, approximately $30 \mathrm{E} 2$ proteins and more than 500 different species of E3 in humans) are mobilized with this cascade system. The set of E3 proteins is highly diverse, because each E3 enzyme usually

\footnotetext{
*1 Laboratory of Frontier Science, Tokyo Metropolitan Institute of Medical Science, Tokyo, Japan.

$\dagger$ Correspondence should be addressed: K. Tanaka, Laboratory of Frontier Science, Tokyo Metropolitan Institute of Medical Science, 3-18-22, Honkomagome, Bunkyo-ku, Tokyo 113-8613, Japan (e-mail: tanaka-kj@igakuken.or.jp).
}

selectively recognizes a protein substrate for ubiquitylation. Furthermore, it should be noted that ubiquitylation is a reversible reaction, because many cysteine-protease and metalloprotease deubiquitylating enzymes (DUBs) are present in the cell. Interestingly, the human genome encodes approximately 95 putative DUBs. ${ }^{3}$ Certain DUBs are responsible for the maturation of ubiquitin from its precursor proteins and products of genes that encode polyubiquitin or ubiquitin fused with ribosomal proteins. Other DUBs function at the initial stage during the breakdown of ubiquitin-tagged proteins to allow ubiquitins to be recycled. The ubiquitin-proteasome system (UPS) controls almost all basic cellular processes - such as progression through the cell cycle, signal transduction, cell death, immune responses, metabolism, protein quality control and development-by degrading short-lived regulatory or structurally aberrant proteins. ${ }^{4)-6)}$ The divergent roles of the UPS have been reported in detail and reviewed comprehensively. ${ }^{1-6)}$ In this review, I provide an overview of the structure and functions of uniquely specified proteasomes. Due to space limitations, I have primarily cited review articles with the exception of particularly important or recently published papers.

\section{26S and 30S Proteasomes}

The proteasome is made up of two subcom- 
Table 1. Subtypes of proteasomes and their regulators

\begin{tabular}{|c|c|}
\hline & Other nomenclature \\
\hline \multicolumn{2}{|l|}{ Catalytic 20S Proteasomes } \\
\hline $\begin{array}{l}\text { Standard (or Constitutive) } \\
\text { Proteasome }\end{array}$ & 20S Core Particle (CP) \\
\hline \multicolumn{2}{|l|}{ Immunoproteasome } \\
\hline \multicolumn{2}{|l|}{ Thymoproteasome } \\
\hline \multicolumn{2}{|l|}{ Testis-specific Proteasome } \\
\hline \multicolumn{2}{|l|}{ Regulators } \\
\hline PA700 & 19S Regulatory Particle (RP) \\
\hline PA200 & Blm10 \\
\hline $\mathrm{PA} 28 \alpha \beta$ & 11S Regulator (REG) \\
\hline \multicolumn{2}{|l|}{ PA28 $\gamma$} \\
\hline \multicolumn{2}{|l|}{ Active Proteasomes } \\
\hline $\begin{array}{l}\text { PA700-CP-PA700 } \\
(19 \mathrm{~S}-20 \mathrm{~S}-19 \mathrm{~S})\end{array}$ & 30S Proteasome \\
\hline PA700-CP (19S-20S) & 26S Proteasome \\
\hline \multicolumn{2}{|l|}{ PA200-CP-PA200 } \\
\hline \multicolumn{2}{|l|}{ PA200-CP } \\
\hline \multicolumn{2}{|l|}{$\begin{array}{l}\text { PA28 } \alpha \beta-\text { CP-PA2 } 28 \alpha \beta \\
(11 \mathrm{~S}-20 \mathrm{~S}-11 \mathrm{~S})\end{array}$} \\
\hline \multicolumn{2}{|l|}{$\mathrm{PA} 28 \alpha \beta-\mathrm{CP}^{*}$} \\
\hline $\mathrm{PA} 28 \alpha \beta-\mathrm{CP}-\mathrm{PA} 700$ & Hybrid Proteasome \\
\hline \multicolumn{2}{|l|}{ PA2 $28 \gamma-\mathrm{CP}-\mathrm{PA} 28 \gamma$} \\
\hline \multicolumn{2}{|l|}{$\mathrm{PA} 28 \gamma-\mathrm{CP}^{*}$} \\
\hline \multicolumn{2}{|l|}{ PA28 $\gamma-\mathrm{CP}-\mathrm{PA} 700^{*}$} \\
\hline \multicolumn{2}{|l|}{ PA200-CP-PA700 ${ }^{* *}$} \\
\hline \multicolumn{2}{|l|}{ PA28 $\alpha \beta-\mathrm{CP}-\mathrm{PA} 200^{*}$} \\
\hline PA28 $\gamma-\mathrm{CP}-\mathrm{PA} 200^{*}$ & \\
\hline
\end{tabular}

plexes: a catalytic core particle (CP; also known as the $20 \mathrm{~S}$ proteasome) and one or two terminal $19 \mathrm{~S}$ regulatory particle(s) (RP) that serves as a proteasome activator with a molecular mass of approximately $700 \mathrm{kDa}$ (called PA700) (Table 1). ${ }^{7)-9)}$ The 19S RP binds to one or both ends of the latent $20 \mathrm{~S}$ proteasome to form an enzymatically active proteasome. The apparent sedimentation coefficient of the active proteasome as determined by densitygradient centrifugation analysis is $26 \mathrm{~S}$ and accordingly the complex is usually referred to as the $26 \mathrm{~S}$ proteasome. Physicochemical analysis, however, has revealed that the correct sedimentation coefficient is approximately $30 \mathrm{~S} .{ }^{10)}$ The size difference is probably due to the attachment of one $19 \mathrm{~S} \mathrm{RP}$ to the $20 \mathrm{~S}$ proteasome to form the so-called $26 \mathrm{~S}$ proteasome, whereas the elongated 30S molecule, which is likely the functional unit in the cell, may include a pair of symmetrically disposed 19S RPs that are attached to both ends of the central portion of the complex (Fig. 1). In this article, however, I will primarily use $26 \mathrm{~S}$ proteasome without distinguishing between these two forms of the proteasome, unless otherwise specified.

As mentioned above, the $26 \mathrm{~S}$ proteasome is a 2.5-MDa multicatalytic degradation machine that contains a 20S CP and one or two 19S RPs, which associate with the termini of the barrel-shaped central particle. The 19S RP serves to recognize ubiquitylated client proteins and is thought to play a role in their unfolding and translocation into the interior of the $20 \mathrm{~S} \mathrm{CP}$, which contains catalytic

\footnotetext{
Abbreviations:

aa: amino acids

AAA-ATPase: ATPase associated with diverse cellular activities

AIRAP: arsenite-inducible proteasomal $19 \mathrm{~S}$ regulatory-associated protein

AIRAPL: AIRAP-like gene

CD: cluster of differentiation

CP: core particle

cTECs: cortical thymic epithelial cells

CTL: cytotoxic T lymphocyte

DSBs: double strand breaks

DUB: deubiquitylating enzyme

EM: electron microscopy

FDA: Food and drug administration

GFP: green fluorescent protein

HbYX: hydrophobic-tyrosine-X

$\mathrm{HCV}$ : hepatitis $\mathrm{C}$ virus

IFN: interferon

MG-132: $N$-carbobenzoxy-leu-leu-leucinal

MHC: major histocompatibility complex
}

\author{
NER: nuclear excision repair \\ Ntn: N-terminal nucleophile \\ PA: proteasome activator \\ PAC: proteasome assembling chaperone \\ PGPH: peptidylglutamyl-peptide hydrolyzing \\ PI: proteasome inhibitor \\ PIP: proteasome-interacting protein \\ POMP: proteasome maturation protein \\ PSI: $N$-carbobenzoxy-L-gamma-t-butyl-L-glutamyl-L-alanyl- \\ L-leucinal \\ REG: 11S regulator \\ $\mathrm{RP}$ : regulatory particle \\ siRNA: small interfering RNA \\ TAP: transporter associated with antigen processing \\ TCR: T cell receptor \\ TOP: thimet oligopeptidase \\ UBA: ubiquitin-associated \\ UBL: ubiquitin-like \\ UPS: ubiquitin-proteasome system \\ Z-L ${ }_{3}$ VS: carboxybenzyl-leucyl-leucyl-leucine vinyl sulfone
}



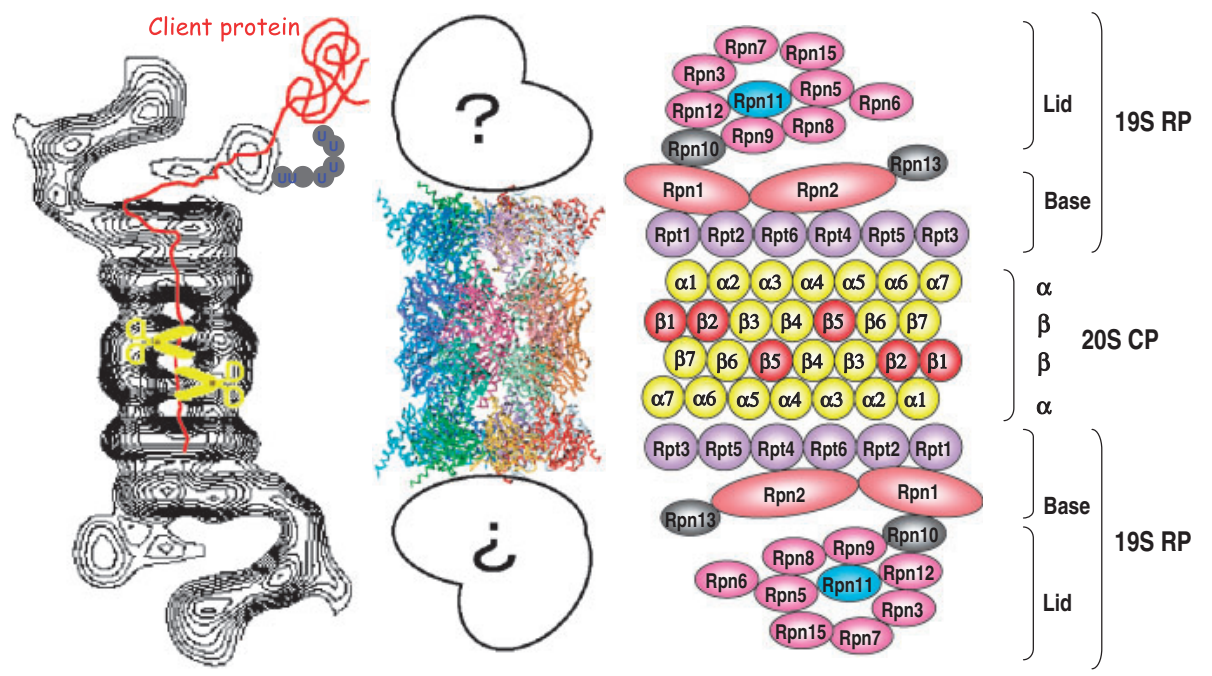

Fig. 1. Schematic diagram of the 26S proteasome. Left panel: Averaged image of the rat 26S proteasome complex based on electron micrographs. Photograph kindly provided by W. Baumeister. U, ubiquitin. Middle panel: The overall tertiary structure of the bovine 20S proteasome (central portion); the structures of the 19S RPs have not yet been determined (the pair of symmetrically disposed terminal structures depicted by question marks). Right panel: Schematic drawing of the subunit structure. CP, core particle (20S proteasome); RP, 19S regulatory particle consisting of the base and lid subcomplexes; Rpn, RP non-ATPase; Rpt, RP triple-ATPase.

threonine residues on the surface of a chamber formed by two $\beta$-rings.

\section{The CP or 20S Proteasome}

The 20S CP (alias 20S proteasome) is well characterized structurally (Fig. 1). It is a wellorganized protein complex with a sedimentation coefficient of $20 \mathrm{~S}$ and a molecular mass of approximately $750 \mathrm{kDa}$. When viewed electron microscopically, the 20S proteasome appears as a cylinder-like structure in various eukaryotes, including yeast and mammals. It forms a packed particle, a result of axial stacking of two outer $\alpha$-rings and two inner $\beta$ rings, which are made up of seven structurally similar $\alpha$ and $\beta$ subunits, respectively; the rings form an $\alpha_{1-7} \beta_{1-7} \beta_{1-7} \alpha_{1-7}$ structure. The $20 \mathrm{~S}$ proteasome plays essentially the same proteolytic roles in all eukaryotes, differing from proteasomes in prokaryotes that mainly consists of homo-hepatmeric $\alpha$ - and $\beta$-rings of the same $\alpha$ and $\beta$ subunits, respectively, i.e., the $\alpha \beta \beta \alpha$ structure. ${ }^{8), 11)}$ Accordingly, the overall structures and functions of the individual subunits are highly conserved among eukaryotic species, except for a specialized form(s) that is associated with adaptive immune responses, which will be described in a later section. Indeed, the yeast (Saccharomyces cerevisiae) and mamma- lian (bovine) $20 \mathrm{~S}$ proteasomes are characterized by the same highly ordered, quaternary structures, as demonstrated by X-ray crystallography. ${ }^{12), 13)}$ The subunits of the 20S proteasome are specifically located within the complex with $\mathrm{C} 2$ symmetry. These subunits are listed in Table 2 .

The three $\beta$-type subunits of each inner ring contain catalytically active threonine residues at their $\mathrm{N}$ termini and show $\mathrm{N}$-terminal nucleophile (Ntn) hydrolase activity, indicating that the proteasome is a threonine protease that does not fall into the known seryl, thiol, carboxyl and metalloprotease families. The $\beta 1, \beta 2$ and $\beta 5$ subunits are associated with caspase-like/PGPH (peptidylglutamyl-peptide hydrolyzing), trypsin-like and chymotrypsin-like activities, respectively, which confer the ability to cleave peptide bonds at the Cterminal side of acidic, basic and hydrophobic amino-acid residues, respectively. Two pairs of these three active sites face the interior of the cylinder and reside in a chamber formed by the centers of the abutting $\beta$-rings. The crystal structure of the 20S proteasome reveals that the center of the $\alpha$-ring is almost completely closed, preventing proteins from penetrating into the inner chamber of the $\beta$-ring that contains the proteolytically active sites. Moreover, the $\mathrm{N}$ termini of the $\alpha$ subunits 
Table 2. Proteasome subunits and proteasome-interacting proteins (PIPs) known to function as auxiliary factors

\begin{tabular}{|c|c|c|c|c|c|c|c|c|c|}
\hline \multirow{2}{*}{ Category } & \multirow{2}{*}{ Subclassification } & \multirow{2}{*}{$\begin{array}{c}\text { Systematic } \\
\text { nomenclature }\end{array}$} & \multirow{2}{*}{ HUGO - } & \multicolumn{2}{|c|}{ Miscellaneous nomenclature } & \multirow{2}{*}{$\begin{array}{c}\text { human (yeast) } \\
\text { amino acids }\end{array}$} & \multirow{2}{*}{ Motif } & \multirow{2}{*}{ Lethality } & \multirow{2}{*}{ Function } \\
\hline & & & & Human & Yeast (budding/fission) & & & & \\
\hline \multirow[t]{19}{*}{$20 \mathrm{~S} \mathrm{CP}$} & $\alpha$ type & $\alpha 1$ & PSMA6 & iota & SCL1, YC7 & (252) 246 & NLS & + & \\
\hline & subunits & $\alpha 2$ & PSMA2 & C3 3 & PRE8, Y7 & (250) 233 & NLS & + & \\
\hline & & $\alpha 3$ & PSMA4 & C9 & PRE9, Y13 & (258) 261 & NLS & - & \\
\hline & & $\alpha 4$ & PSMA7 & $\mathrm{C} 6$ & PRE6 & (254) 248 & NLS & + & \\
\hline & & $\alpha 5$ & PSMA5 & zeta & PUP2, DOA5 & (260) 241 & & + & \\
\hline & & $\alpha 6$ & PSMA1 & $\mathrm{C} 2$ & PRE5 & (234) 263 & & + & \\
\hline & & $\alpha 7$ & PSMA3 & $\mathrm{C} 8$ & PRE10, YC1 & (288) 254 & & + & \\
\hline & & $\alpha 8$ & PSMA8 & & - & 256 & & & \\
\hline & $\beta$ type & $\beta 1$ & PSMB6 & $\mathrm{Y}$, delta & PRE3 & (19+196) $34+205$ & Ntn & + & Caspase-like \\
\hline & subunits & $\beta 2$ & PSMB7 & $\mathrm{Z}$ & PUP1 & $(29+232) 43+234$ & Ntn & + & Trypsin-like \\
\hline & & $\beta 3$ & PSMB3 & $\mathrm{C} 10$ & PUP3 & (205) 205 & & + & \\
\hline & & $\beta 4$ & PSMB2 & $\mathrm{C} 7$ & PRE1 & (198) 201 & & + & \\
\hline & & $\beta 5$ & PSMB5 & $\mathrm{X}, \mathrm{MB} 1$, epsilon & PRE2, DOA3 & $(75+212) 59+204$ & Ntn & + & Chymotrypsin-like \\
\hline & & $\beta 6$ & PSMB1 & $\mathrm{C} 5$ & PRE7 & $(19+222) 28+213$ & & + & \\
\hline & & $\beta 7$ & PSMB4 & N3, beta & PRE4 & $(33+233) 45+219$ & & + & \\
\hline & & $\beta 1 \mathrm{i}$ & PSMB9 & LMP2, RING12 & - & $20+199$ & Ntn & $(-)$ & Caspase-like \\
\hline & & $\beta 2 \mathrm{i}$ & PSMB10 & MECL1, LMP10 & - & $39+234$ & Ntn & $(-)$ & Trypsin-like \\
\hline & & $\beta 5 \mathrm{i}$ & PSMB8 & LMP7, RING10 & - & $72+204$ & Ntn & $(-)$ & Chymotrypsin-like \\
\hline & & $\beta 5 \mathrm{t}$ & PSMB11 & & - & $44+251$ & Ntn & $(-)$ & Chymotrypsin-like \\
\hline \multirow{14}{*}{$\begin{array}{c}\text { PA700 } \\
(19 S \text { RP) }\end{array}$} & ATPase & Rpt1 & PSMC2 & S7, Mss1 & YTA3, CIM5 & (467) 433 & $\mathrm{AAA}$ & + & ATPase \\
\hline & subunits & Rpt2 & PSMC1 & $\mathrm{S} 4, \mathrm{p} 56$ & YTA5/mts2 & (437) 440 & $\mathrm{AAA}, \mathrm{HbYX}$ & $+(-)$ & ATPase, Gate-opning \\
\hline & & Rpt3 & PSMC4 & S6, Tbp7, P48 & YTA2 & (428) 418 & AAA, HbYX & $+(+)$ & ATPase, Gate-opning \\
\hline & & Rpt4 & PSMC6 & S10b, p42 & SUG2, PCS1, CRL13 & (437) 389 & AAA & + & ATPase \\
\hline & & Rpt5 & PSMC3 & $\mathrm{S} 6^{\prime}, \mathrm{Tbp} 1$ & YTA1 & (434) 439 & $\mathrm{AAA}, \mathrm{HbYX}$ & $+(+)$ & ATPase, Gate-opning \\
\hline & & Rpt6 & PSMC5 & $\mathrm{S} 8, \mathrm{p} 45$, Trip1 & SUG1, CRL3, CIM3/let1 & (405) 406 & AAA & + & ATPase \\
\hline & non-ATPase & Rpn1 & PSMD2 & S2, p97 & HRD2, NAS1/mts4 & (993) 908 & $\mathrm{PC}$ & + & PIPs scaffold \\
\hline & subunits & Rpn2 & PSMD1 & $\mathrm{S} 1, \mathrm{p} 112$ & SEN3 & (945) 953 & PC, NLS & + & PIPs scaffold \\
\hline & & Rpn3 & PSMD3 & $\mathrm{S} 3, \mathrm{p} 58$ & SUN2 & (523) 534 & PCI, PAM & + & \\
\hline & & Rpn5 & PAMD12 & $\mathrm{p} 55$ & NAS5 & (445) 456 & PCI & + & \\
\hline & & Rpn6 & PSMD11 & $\mathrm{S} 9, \mathrm{p} 44.5$ & NAS4 & (434) 422 & PCI, PAM & + & \\
\hline & & Rpn7 & PSMD6 & $\mathrm{S} 10 \mathrm{a}, \mathrm{P} 44$ & & (429) 389 & PCI & + & \\
\hline & & Rpn8 & PSMD7 & $\mathrm{S} 12, \mathrm{p} 40, \mathrm{MOV} 34$ & NAS3 & (338) 324 & MPN & + & \\
\hline & & Rpn9 & PSMD13 & $\mathrm{S} 11, \mathrm{p} 40.5$ & $\mathrm{NAS} 7 / \mathrm{mts} 1$ & (393) 376 & PCI & - & \\
\hline
\end{tabular}




\begin{tabular}{|c|c|c|c|c|c|c|c|c|c|}
\hline \multirow{2}{*}{ Category } & \multirow{2}{*}{ Subclassification } & \multirow{2}{*}{$\begin{array}{c}\text { Systematic } \\
\text { nomenclature }\end{array}$} & \multirow{2}{*}{ HUGO } & \multicolumn{2}{|c|}{ Miscellaneous nomenclature } & \multirow{2}{*}{$\begin{array}{c}\text { human (yeast) } \\
\text { amino acids }\end{array}$} & \multirow{2}{*}{ Motif } & \multirow{2}{*}{ Lethality } & \multirow{2}{*}{ Function } \\
\hline & & & & Human & Yeast (budding/fission) & & & & \\
\hline & & Rpn10 & PSMD4 & S5a, Mbp1 & SUN1, MCB1/pus1 & (268) 377 & UIM, VWA & $-(+)$ & Ub receptor \\
\hline & & Rpn11 & PSMD14 & S13, Poh1 & MPR1/pad1, mts5 & (306) 310 & MPN, JAMM & + & DUB \\
\hline & & Rpn12 & PSMD8 & $\mathrm{S} 14, \mathrm{p} 31$ & NIN1/mts3 & (274) 257 & PCI & + & \\
\hline & & Rpn13 & ADRM1 & ADRM1 & DAQ1 & (156) 407 & Pru & - & $\begin{array}{l}\text { Ub receptor, } \\
\text { Uch } 37 \text { recruit }\end{array}$ \\
\hline & & Rpn15 & SHFM1 & DSS1, SHFM1 & SEM1 & (89) 70 & & - & \\
\hline & & & PSME1 & $\mathrm{PA} 28 \alpha, \mathrm{REG} \alpha$ & - & 249 & & $(-)$ & PSM activator \\
\hline \multirow{2}{*}{ (11S REG) } & & & PSME2 & $\mathrm{PA} 28 \beta, \mathrm{REG} \beta$ & - & 239 & & $(-)$ & PSM activator \\
\hline & & & PSME3 & $\mathrm{PA} 28 \gamma, \mathrm{REG} \gamma, \mathrm{Ki}$ & - & 254 & & $(-)$ & PSM activator \\
\hline PA200 & & & PSME4 & PA200, TEMO & BLM10 & (2143) 1843 & HEAT, ARM & $(-)$ & PSM activator \\
\hline PI31 & & & PSMF1 & & - & 271 & Proline-rich & & PSM inhibitor \\
\hline \multirow{6}{*}{$\begin{array}{l}\text { Assembling } \\
\text { chaperones }\end{array}$} & & & & & & & & & \\
\hline & & hUmp1 & POMP & Proteassemblin & UMP1 & (148) 141 & & $-(+)$ & PSM formation \\
\hline & & $\mathrm{PAC} 1$ & PSMG1 & & Pba1, Poc1 & (276) 288 & & $-(+)$ & PSM formation \\
\hline & & $\mathrm{PAC} 2$ & PSMG2 & & Pba2, ADD66, Poc2 & (267) 264 & & - & PSM formation \\
\hline & & PAC3 & PSMG3 & & Pba3, Dmp2, Poc3 & (179) 122 & & - & PSM formation \\
\hline & & $\mathrm{PAC} 4$ & PSMG4 & & Pba4, Dmp1, Poc4 & (148) 123 & & - & PSM formation \\
\hline \multirow[t]{13}{*}{ PIPs } & & & PSMD5 & $\mathrm{S} 5 \mathrm{~b}, \mathrm{p} 50.5$ & - & 504 & ARM & & \\
\hline & & Rpn4 & & - & SON1, UFD5 & $(531)$ & Zn finger & - & $\begin{array}{l}\text { PSM gene } \\
\text { transcription }\end{array}$ \\
\hline & & Rpn14 & PAAF1 & FLJ11848 & YGL004C & (417) 392 & WD40, G-beta & - & PSM inhibitor \\
\hline & & & PSMD9 & p27 & NAS2 & (220) 223 & PDZ & - & PSM modulator \\
\hline & & & PSMD10 & p28, gankyrin & NAS6 & (228) 226 & ANK & - & \\
\hline & & KIAA0368 & KIAA0368 & ECM29 & Ecm29 & (1868) 1870 & HEAT & - & PSM stabilizer \\
\hline & & USP14 & USP14 & & Ubp6/ubp6 & (499) 494 & & - & DUB \\
\hline & & UCHL5 & HCHL5 & Uch37 & -/uch2 & 329 & & & DUB \\
\hline & & UBE3C & UBE3C & KIAA10 & Hul5 & (910) 1083 & RING & & Ub ligase \\
\hline & & & UBE3A & E6AP & - & 852 & HECT & & Ub ligase \\
\hline & & & PARK2 & Parkin & - & 465 & UBL, RING & $(-)$ & Ub ligase \\
\hline & & & RAD23A/B & $\mathrm{hH} 23 \mathrm{~A} / \mathrm{B}$ & $\operatorname{Rad} 23$ & (398) 363/409 & UBL, UBA & - & Shuttling factor \\
\hline & & & UBQLN1/2 & hPLIC-1/2 & DSK2 & (373) 589/624 & UBL, UBA & - & Shuttling factor \\
\hline
\end{tabular}

(+): Lethal (mouse), (-): Non-lethal (mouse), +: Lethal (yeast), -: Non-lethal (yeast), —: No orthologue, AAA: ATPase associated with diverse cellular activities, ANK: ankyrin repeats, ARM: Armadillo repeats, Amino acids ( $\beta$ subunit): Propeptide + mature protein, DUB: Deubiquitylating enzyme, HECT: a domain homologous to the E6AP carboxyl terminus, HUGO: Human Genome Organization, MPN: Mpr1, Pad1 N-terminal, NLS: Nuclear localization signal, Ntn: N-terminal nucleophile hydrolase, PAC: Proteasome assembling chaperone, PAM: PCI associated module, PC: proteasome/cyclosome repeat, HbYX: hydrophobic-tyrosine-X, PCI: proteasome, COP9,eIF3, PDZ: PSD-95/DLG/ZO-1, PIPs: Proteasome interacting proteins, PSM: Proteasome, Pru: Pleckstrin-like receptor for ubiquitin, RING: Ring finger, UBA: Ubiquitin associated, UBL: Ubiquitin-like, UIM: Ubiquitin Interacting Motif, Ub: Ubiquitin, VWA: von Willebrand factor type A. 
form an additional physical barrier for access to the active sites. ${ }^{14)}$ Thus, the $20 \mathrm{~S}$ proteasome is latent in cells; substrates are able to access the active sites only after passing through the narrow opening at the center of the $\alpha$-rings.

The 20S proteasome processively degrades client proteins, generating oligopeptides ranging in length from 3 to 15 amino-acid residues. The resulting peptide products are subsequently hydrolyzed to amino acids by oligopeptidases and/or amino-carboxyl peptidases. One such enzyme is the metalloendopeptidase thimet oligopeptidase (TOP), which associates with the $26 \mathrm{~S}$ proteasome (our unpublished results) and displays an efficient hydrolytic activity in the soluble fraction of the cells. ${ }^{15)}$ Of note, there is evidence to suggest that unfolded proteins are generated in response to stressors. For example, proteins damaged by oxidation or intrinsically unstructured proteins (also known as natively unfolded proteins) are degraded directly by the $20 \mathrm{~S}$ proteasome. The mechanisms that control the gate opening of the closed $\alpha$-ring, however, are poorly understood, although the binding of denatured proteins to the $\alpha$-ring seem to help open the gate. ${ }^{16), 17)}$ While this process has been examined in vitro, it is not clear at this stage whether the 20S proteasome itself is responsible for proteolysis in vivo without facilitation by other activator protein(s).

\section{The RP or PA700}

The enzymatically active proteasome is generally capped on either or both ends of the central $20 \mathrm{~S}$ proteasomal core by regulatory proteins (Table 1 ). The RP recognizes client proteins marked by polyubiquitin chains, removes the chain and entraps the protein moiety, unfolds the substrate proteins, opens the $\alpha$-ring, and transfers the unfolded substrates into the CP for destruction (Fig. 2). The 19S RP comprises approximately 20 different subunits that can be subclassified into two groups: Regulatory particle of triple-ATPase (Rpt) subunits and Regulatory particle of non-ATPase (Rpn) subunits, both of which contain multiple proteins with molecular masses ranging from 10 to $110 \mathrm{kDa}$. The following is a brief description of the 19S RP (alias PA700), which comprises two subcomplexes: the lid and the base. ${ }^{18), 19)}$

3.1 The lid subcomplex. The lid complex is composed of at least nine non-ATPase subunits:

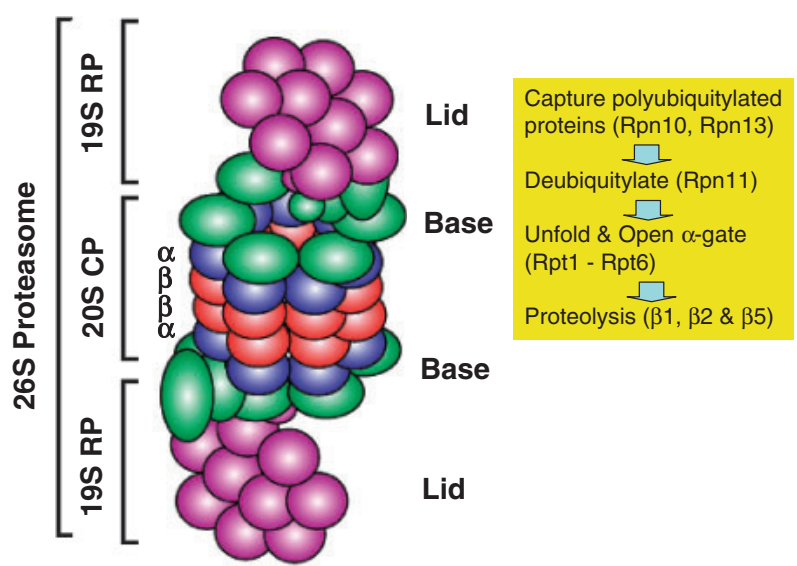

Fig. 2. Schematic diagram of proteolysis by the $26 \mathrm{~S}$ proteasome. Positions of subunits indicated (i.e., Rpn10, Rpn13, Rpn11, Rpt1-Rpt6, $\beta 1, \quad \beta 2$ and $\beta 5$ are represented in Fig. 1. The $\beta 1, \beta 2$ and $\beta 5$ subunits are associated with caspase-like, trypsin-like and chymotrypsin-like activities, respectively (for details, see text).

Rpn3, Rpn 5, Rpn6, Rpn 7, Rpn 8, Rpn 9, Rpn 11, Rpn 12 and Rpn 15 (Fig. 1). The main function of the lid is to deubiquitylate the captured substrates, a process in which the metalloisopeptidase Rpn11 functions to recycle the ubiquitins (Fig. 2). ${ }^{20)}$ Indeed, Rpn11 DUB cleaves the polyubiquitin chain at a proximal site; this chain is further cleaved into monomeric ubiquitins by other DUBs. In addition, in mammalian cells, two other DUBs that are physically associated with the base complex cleave the ubiquitin moiety at a distal site. Usp14 (yeast UBP6) is associated with Rpn121) and Uch37 binds to the C-terminal domain of Rpn2-bound Rpn13; i.e., Uch37 associates with the base via Rpn13. ${ }^{22), 23)}$ Intriguingly, deubiquitylation by Uch37 is activated by proteasome binding, which is also involved in the editing of polyubiquitin chains. In addition, the yeast Ubp6 is induced by ubiquitin deficiency although ubiquitin stress does not upregulate proteasome abundance. Namely the enhanced loading of proteasomes with Ubp6 alters proteasome function, implying a dual role for Ubp6 in regulating ubiquitin levels and proteasome function. ${ }^{24}$ The functions of most of the other subunits in the lid, however, have yet to be elucidated.

3.2 The base subcomplex. The base complex is composed of six homologous AAA-ATPase subunits, (Rpt1-Rpt6) and four non-ATPase subunits (Rpn1, Rpn2, Rpn10 and Rpn13 Fig. 1). The base complex of proteasomes has three functional 
roles: capturing client proteins via ubiquitin recognition, promoting substrate unfolding and opening the channel in the $\alpha$-ring (Fig. 2). Recently, a novel functional unit within the lid complex, comprising two subunits, Rpn1 and Rpn2 was proposed; i.e., Rpn2 interfaces with the 20S, whereas Rpn1 sits atop Rpn2, serving as a docking site for a substraterecruitment factor. ${ }^{25)}$ The lid ATPases encircle the Rpn1-Rpn2 stack, covering the remainder of the 20S surface. Both Rpn1-Rpn2 and the ATPases are required for substrate translocation and gating of the proteolytic channel.

Rpn10 and Rpn13 function as integral ubiquitin receptors and efficiently trap polyubiquitylated substrates. Rpn10 achieves this function via a Cterminal ubiquitin-interacting motif (UIM). ${ }^{26)}$ More recently, Rpn13 was identified as a second ubiquitin receptor. ${ }^{27-29)}$ The N-terminal domain of Rpn13 shows no similarity to known ubiquitin-binding motifs, but instead contains the novel 'pleckstrinlike receptor for ubiquitin' (Pru) domain. The Pru domain in human Rpn13 shows a high affinity for diubiquitin. It is interesting that the $\mathrm{C}$-terminal domain of Rpn13 produces the DUB activity, which may collaborate with the N-terminal ubiquitin receptor within the same molecule to facilitate proteolysis. It is possible that Rpn13 is not an intrinsic proteasome subunit, because the $26 \mathrm{~S}$ proteasome, which does not contain Rpn13, exists to a lesser extent in mammalian cells. ${ }^{22)}$ In addition, although Rpt5 has been reported to bind polyubiquitylated proteins in vitro, ${ }^{30)}$ there is no direct evidence that it functions as a ubiquitin receptor in the cell.

In addition to the intrinsic ubiquitin receptors, there are several extrinsic UBL (ubiquitin-like)UBA (ubiquitin-associated) ubiquitin receptors, such as Rad23, Dsk2 and Ddi1 (Table 2). ${ }^{31), 32)}$ In higher eukaryotes, these UBL-UBA proteins interact with the $26 \mathrm{~S}$ proteasome via their UBL domains. Indeed, the UBL domain can bind directly the Rpn10, which also functions as an acceptor site of polyubiquitylated proteins due to the UBA domain. Thus, UBL-UBA proteins also function cooperatively with several intrinsic receptors to recognize polyubiquitylated proteins. ${ }^{33)}$ In addition, the p62/SQSTM1, which contains Nterminal PB1 whose tertial structure resembles UBL, C-terminal UBA domains and VCP (also known as CDC48 or p97 ATPase), also binds both the proteasome and polymerized ubiquitin chains, thereby localizing client proteins to the $26 \mathrm{~S}$ proteasome. ${ }^{34), 35)}$ It was reported recently, however, that p62 functions as a key factor to direct ubiquitylated proteins to autophagy (i.e., self-destruction through the lysosomal machinery). ${ }^{36), 37)}$ It is worth noting that Rpn1 (and Rpn2) is also responsible for acceptance of various UBL proteins, ${ }^{38)}$ similar to Rpn10.

Emerging evidence has provided significant insights into the roles of the ATPases in the base subcomplex. Substrates access the catalytic sites through the central pore in the $\alpha$-rings. Free CP exists in an autoinhibited form in which the $\mathrm{N}$ termini of the $\alpha$ subunits create a gate that blocks substrate entry. The base contains six ATPase subunits (Rpt1-6) organized into a hexameric ring that facilitates the opening of the gate and allows the substrate to reach the catalytic sites. How proteasomal ATPases promote the gate opening in proteasomes remains a long-standing question. 20S proteasomes are activated following opening of the gate by the proteasome activators PA700 (i.e., the base of 19S RP) and PA28 (Ref. 39 see below for details). The PA28-mediated mechanism involved in gate control is well established. PA26 from Trypanosoma brucei, which is similar to mammalian PA28, is a homoheptameric complex. The crystal structure of the PA26-20S proteasome complex shows that PA26 binds to 20S proteasomes by inserting its $\mathrm{C}$ terminus into the intersubunit pocket between adjacent $\alpha$ subunits. ${ }^{40)}$ In addition, the "activation loop" domain of PA26 stabilizes the open-gate conformation. Interestingly, activation of $20 \mathrm{~S}$ proteasomes by PAN, an archaeal homohexameric ATPase complex that is related to eukaryotic 19S ATPases, also requires the C-terminal residues of PAN. This suggests that the proteasomal ATPases and PA26 employ similar mechanisms to open the proteasome gate.

The archaeal PAN ATPase complex and the three 19S ATPase subunits each contain a conserved C-terminal hydrophobic-tyrosine-X (HbYX) motif required for gate opening. The $\mathrm{C}$ termini of the PAN molecules are inserted into the $20 \mathrm{~S}$ pockets to induce gate opening through a mechanism that resembles a key and a lock. ${ }^{41), 42)}$ Among the six 19S ATPases, only Rpt2, Rpt3 and Rpt5 contain the HbYX motif, and it is clear that multiple HbYX motifs from the three subunits 
specifically facilitate the gate opening. One unresolved question is how the 6-fold symmetric ATPase ring associates with the 7 -fold symmetric $20 \mathrm{~S}$ $\alpha$-ring to stabilize the open-gate conformation. Because only 2-4 subunits of the hexameric ATPase structure are thought to simultaneously bind ATP, not all of proteasomal ATPase subunits synchronously work to open the gate. Thus, only a subset of the C-terminal ends of the ATPase subunits inserts into the 20S pockets, which may occur sequentially to stabilize the open gate through an apparent "wobbling" of the proteasomal ATPases. Alternatively, the subunits may show different ATPase rates; e.g., Rpt2 and Rpt5 may be always bound to ATP to stabilize the open-gate conformation, whereas the remaining ATPase subunits may move dynamically through the ATPase cycle to promote protein unfolding. In this model, the $26 \mathrm{~S}$ proteasome is rather a stable complex and two or three ATPase subunits simultaneously bind the $20 \mathrm{~S}$ pockets to open the gate. It is noteworthy that similar to the PA28-mediated mechanism, ATP binding is sufficient for gate opening without requiring ATP hydrolysis. ${ }^{43}$

The base subcomplex ATPases are required for not only $\alpha$-ring channel opening but also substrate unfolding. Because the protein-degrading sites lie inside the 20S CP and are accessible only through the narrow channel, substrate proteins must be unfolded before they can reach the active sites in the $\beta$-ring chamber. Although details of the underlying ATP-dependent mechanism are still largely unknown, it is clear that the base subcomplex ATPases play a central role in substrate unfolding, through a process that requires ATP hydrolysis. ${ }^{44)}$ Intriguingly, the base complex shows a chaperone activity in vitro that can cause refolding of unfolded proteins in the presence of ATP, but neither in the presence of ADP nor the absence of ATP. ${ }^{45)}$

\section{PA28 and Hybrid Proteasome}

PA28 or the 11S regulator (REG) was identified as another protein activator of the latent $20 \mathrm{~S}$ proteasome (Table 1). ${ }^{39)}$ Electron microscopic examination revealed that PA28 forms conical caps by associating with both ends of the central 20S CP. ${ }^{7)}$ PA28 complexes are composed of three structurally-related members designated $\alpha, \beta$ and $\gamma$; their primary structures display approximately $50 \%$ homology. ${ }^{46)}$ Whereas the PA28 $\alpha$ and PA28 $\beta$ assemble into hetero-oligomeric complexes with alternating $\alpha$ and $\beta$ subunits, the PA28 $\gamma$ appears to form homopolymeric complexes. Immunofluorescence analysis revealed that both PA28 $\alpha$ and PA2 $8 \beta$ are located mainly in the cytoplasm, whereas PA28 $\gamma$ is located predominantly in the nucleus outside of the nucleolus. ${ }^{47)} \mathrm{X}$-ray crystallographic analysis of recombinant $\mathrm{REG} \alpha$ (PA28 $\alpha$ ) revealed a heptameric complex. ${ }^{48)}$ Whether PA28 $\alpha$ and PA28 $\beta$ form heteroheptameric (i.e., $\alpha 3 \beta 4$ or $\alpha 4 \beta 3$ ) complexes in cells, however, requires further investigation.

4.1 The PA28 $\boldsymbol{\beta} \boldsymbol{\beta}$ complex. The PA28 protein stimulates all of the peptidase activities of the $20 \mathrm{~S}$ proteasome without affecting the destruction of large protein substrates, even if the proteins have already been polyubiquitylated. Thus, PA28 does not play a central role in the initial cleavage of protein substrates in cells. It presumably has a stimulating effect on the degradation of intermediate-size polypeptides that are generated by the $26 \mathrm{~S}$ proteasome, implying that the $26 \mathrm{~S}$ proteasome and the PA28-proteasome complex may function sequentially or cooperatively.

To assess the precise role of the PA28 $\alpha / \beta$ complex in vivo, we generated mice lacking both the PA28 $\alpha$ and PA28 $\beta$ genes. ${ }^{49)}$ No obvious gross abnormalities were observed in the mutant mice. Available evidence indicates that the proteasome functions as a processing enzyme responsible for the generation of major histocompatibility complex (MHC) class I ligands, which are essential for the initiation of cell-mediated immunity in vertebrates. ${ }^{50)-52)}$ Intriguingly, the immunomodulatory cytokine interferon (IFN)- $\gamma$ induces overexpression of PA28 $\alpha$ and PA28 $\beta .{ }^{46)}$ In addition, IFN- $\gamma$ also overexpresses the majority of proteins related to the MHC class I ligand presentation pathway, such as MHCs and transporter associated with antigen processing (TAP); thus, it is plausible that PA28 $\alpha / \beta$ contributes to efficient production of cytotoxic T lymphocyte (CTL) epitopes. Although splenocytes from PA28 $\alpha^{-/-} \mathrm{PA} 28 \beta^{-/-}$mice displayed no apparent defects in the processing of ovalbumin and normal immune responses against infection with influenza A virus, they almost completely lacked the ability to process a melanoma antigen TRP2-derived peptide. These findings indicate that $\mathrm{PA} 28 \alpha / \beta$ is not required for antigen presentation in general, but instead plays an 
essential role in the processing of certain antigens. ${ }^{49)}$

4.2 The PA28 $\gamma$ complex. The function of PA28 $\gamma$, which is not involved in the processing of intracellular antigens, remains largely unknown. To investigate the roles of PA28 $\gamma$ in vivo, we generated mice lacking the PA28 $\gamma$ gene. ${ }^{53)}$ PA28 $\gamma$-deficient mice were born without appreciable abnormalities in any of the examined tissues, but their growth after birth was retarded compared with that of wild-type mice. We also investigated the effects of PA28 $\gamma$ deficiency in vitro using cultured embryonic fibroblasts; cells lacking PA28 $\gamma$ were larger and displayed a lower saturation density than their wild-type counterparts. Neither the expression of PA28 $\alpha / \beta$ nor the subcellular localization of $\mathrm{PA} 28 \alpha / \beta$ was affected in the PA28 $\gamma^{-/-}$cells. These results indicate that $\mathrm{PA} 28 \gamma$ functions as a regulator of cell proliferation and body growth in mice and demonstrates that neither PA28 $\alpha$ nor PA28 $\beta$ compensates for the PA28 $\gamma$ deficiency.

Recently, numerous reports have addressed the importance of PA28 $\gamma$ in nuclear proteolysis. This protein contributes to the turnover of p53 via MDM2-mediated proteasomal degradation. The polymer form of PA28 $\gamma$ facilitates the physical interaction between MDM2 and p53, promoting MDM2-dependent ubiquitylation and subsequent proteasomal degradation of p53; this process limits p53 accumulation, and thereby inhibits apoptosis after DNA damage. ${ }^{54}$ These findings indicate the involvement of PA28 $\gamma$ in apoptosis and cell proliferation. In addition, PA28 $\gamma$ promotes proteasome-mediated degradation of steroid receptor coactivator-3 (SRC-3), which is encoded by an oncogene that is frequently amplified and overexpressed in breast cancers, highlighting an alternative proteasome-directed degradation mechanism, independent of $19 \mathrm{~S} \mathrm{RP.}{ }^{55)}$ Moreover, PA28 $\gamma$ also enhances the degradation of the cell-cycle regulator $\mathrm{p} 21^{\mathrm{Cip} 1}$, independent of ubiquitylation. ${ }^{56)}$ The role of $\mathrm{PA} 28 \gamma$ in cell-cycle regulation may extend beyond its effect on p21, because $\mathrm{p} 16^{\mathrm{INK} 4 \mathrm{~A}}$ and p19Arf also bind to PA28 $\gamma$ and are stabilized in PA28 $\gamma$-deficient cells. ${ }^{57)}$ In addition, PA28 $\gamma$ is localized in the nucleus in interphase cells and on chromosomes in telophase cells, suggesting a role in mitotic progression. This conclusion is supported by the marked aneuploidy (chromosomal gain and loss), supernumerary centrosomes and multipolar spindles observed in the fibroblasts of PA28 $\gamma$ deficient mice. ${ }^{58)}$ These findings underscore a previously uncharacterized function of PA28 $\gamma$ in centrosomes and chromosomal stability. The discovery that PA28 $\gamma$ controls cell-cycle regulators is consistent with a previous study that described body growth retardation of $\mathrm{PA} 28 \gamma$-deficient mice. ${ }^{59)}$ Finally, PA28 $\gamma$ plays a crucial role in the development of liver pathology induced by hepatitis $\mathrm{C}$ virus (HCV) infection, because knocking out of PA28 $\gamma$ gene induced $\mathrm{HCV}$ core protein accumulation in hepatocyte nuclei of HCV core gene transgenic mice and disrupted the development of both hepatic steatosis and hepatocellular carcinoma. ${ }^{60)}$ Thus, PA28 $\gamma$ appears to play a diverse set of functions in mammals.

4.3 The hybrid proteasome. Immunoprecipitation analysis revealed that the PA28 and PA700 activators simultaneously bind to the $20 \mathrm{~S}$ proteasome; PA28 and PA700 rings bind at opposite ends of the 20S particle, forming the PA700-20S-PA28 complex (Table 1). This complex has been named the "hybrid proteasome" ${ }^{61)}$ Because electron microscopic analysis revealed PA28 and PA700 occupy the same site on the $20 \mathrm{~S} \mathrm{CP}$ and the $26 \mathrm{~S}$ proteasome, respectively, it is surprising that both activators can associate with the same $20 \mathrm{~S}$ proteasome in opposite orientations. ${ }^{62), 63)}$

The hybrid proteasome seems to contribute to efficient proteolysis; intact substrate proteins may be first recognized by PA700 and then fed into the cavity of the 20S proteasome, which shows markedly enhanced cleavage activity in the presence of the PA28 $\alpha \beta$ complex. Indeed, this complex catalyzes ATP-dependent degradation of ornithine decarboxylase (ODC) without ubiquitylation, although it does require antizyme, an ODC inhibitory protein, as does the $26 \mathrm{~S}$ proteasome. ${ }^{64)}$ Intriguingly, IFN- $\gamma$ appreciably enhances the expression of PA2 $2 \alpha \beta$ and consequently promotes the formation of hybrid proteasomes, implying that this complex could be also responsible for the immunological processing of intracellular antigens. Moreover, the hybrid proteasome enhances the hydrolysis of small peptides and generates a pattern of peptides different from those generated by the $26 \mathrm{~S}$ proteasome, without altering the mean product length. ${ }^{62)}$ Presumably, this change in the peptide profile accounts for the capacity of PA28 to enhance antigen presentation. The existence of hybrid 
proteasomes may explain the physiological importance of PA28 $\alpha \beta$ and/or PA28 $\gamma$ as described in previous sections. Therefore, it is plausible that the 26S and hybrid ATP-dependent proteasomes both contribute to the proteolytic pathway in mammalian cells.

\section{PA200 or Blm10}

Yeast Blm10 (formally Blm3 and equivalent to mammalian PA200) is reported to regulate proteasome assembly and/or proteolytic activity, although there are discrepant reports about its precise roles. ${ }^{65), 66)}$ Blm10 was identified in proteasome precursors purified with Ump1 in yeast. Blm10-decicient cells grew apparently normal under normal conditions but the turnover of Ump1 as well as the processing of $\beta 5$ were accelerated, suggesting a role in preventing premature formation of $20 \mathrm{~S}$ proteasomes. ${ }^{67)}$ On the other hand, the combination of Blm10 deletion and $\beta 7 \mathrm{C}$-terminal truncation resulted in severe impairment of proteasome activity and $\beta 2$ processing, indicating that Blm10 promotes proteasome maturation, presumably by stabilizing nascent $20 \mathrm{~S}$ proteasomes. ${ }^{68)}$ The discrepancy in the two paradoxical roles of Blm10 has not yet been explained.

Interestingly, the Blm10-CP-RP complex (i.e., PA200-20S-PA700 in Table 1) is found predominantly. Electron microscopy (EM) studies have shown that Blm10 has a highly elongated, curved structure, and adapts to the end of the CP cylinder, where it is properly positioned to activate the autoinhibited CP (i.e., closed-gate conformation) by opening the axial channel into its proteolytic chamber. ${ }^{66)}$ In contrast, cryo-electron micrographs of the singly bound complex, PA200, shows an asymmetric dome-like structure with major and minor lobes. ${ }^{69), 70)}$ PA200 makes contact with all $\alpha$ subunits except $\alpha 7$, and this interaction induces the opening of the axial channel through the $\alpha$-ring, indicating that the activation mechanism of PA200 is expressed via its allosteric effects on the CP, perhaps facilitating release of digestion products or the entrance of substrates. It was proposed recently that whereas the single-capped Blm10-CP shows peptide hydrolysis activity, the peptide hydrolysis activity is repressed in double-capped Blm10-CPBlm10, suggesting that that Blm10 distinguishes between gate conformations and regulates the activation of $\mathrm{CP}{ }^{71)}$
On the other hand, PA200 was also identified as a broadly expressed nuclear protein that activates proteasomal hydrolysis of peptides, but not proteins. ${ }^{65)}$ In addition, in response to ionizing radiation, PA200 forms alternative hybrid proteasomes with PA200 and PA700 (i.e., PA200-CPPA700) that accumulate on chromatin, leading to an increase in proteolytic activity, preferentially cleavage after acidic residues in vitro. Importantly, cells with PA200-knockdown by small interfering RNA (siRNA) show genomic instability and reduced survival after exposure to ionizing radiation, suggesting a unique role for PA200 in genomic stability that is likely mediated through its ability to enhance postglutamyl cleavage by proteasomes. ${ }^{72)}$ However, PA200-deficient embryonic stem cells do not exhibit increased sensitivity to either ionizing radiation or bleomycin, implying that it is not essential for the repair of DNA DSBs generated in these experimental settings. ${ }^{73)}$ Intriguingly, PA200 knockout mice are viable and exhibit no gross developmental abnormalities, but loss of PA200 led to a marked reduction in male, but not female, fertility, suggesting an important nonredundant function during spermatogenesis.

\section{PI31}

PI31, a previously described inhibitor of $20 \mathrm{~S}$ proteasomes, prevents the activation of the proteasome by each of two proteasome regulatory proteins, PA700 and PA28, suggesting that it plays an important role in control of proteasome function. ${ }^{74}$ PI31 is a proline-rich protein, particularly within its carboxyl-terminal half where $26 \%$ of the amino acids are proline, which appears to have an extended secondary structure. Proteasome inhibition is conferred by the proline-rich domain of PI31. However, it also is reported that PI31 represents a cellular regulator of proteasome formation and of proteasome-mediated antigen processing, based on the observation that PI31 selectively interferes with the maturation of immunoproteasome precursor complexes. ${ }^{75)}$ Surprisingly, recent studies reported that PI31 is structurally related to Fbxo7, the substraterecognition component of the $\mathrm{SCF}^{\mathrm{Fbxo}} \mathrm{E} 3$ ligase. ${ }^{76)}$ PI31 was identified as an Fbxo7-Skp1 binding partner whose interaction requires an N-terminal domain present in both proteins referred to as the FP (Fbxo7/PI31) domain. The PI31 FP domain mediates heterodimerization of $\mathrm{SCF}^{\mathrm{Fbxo}}$ and PI31. 


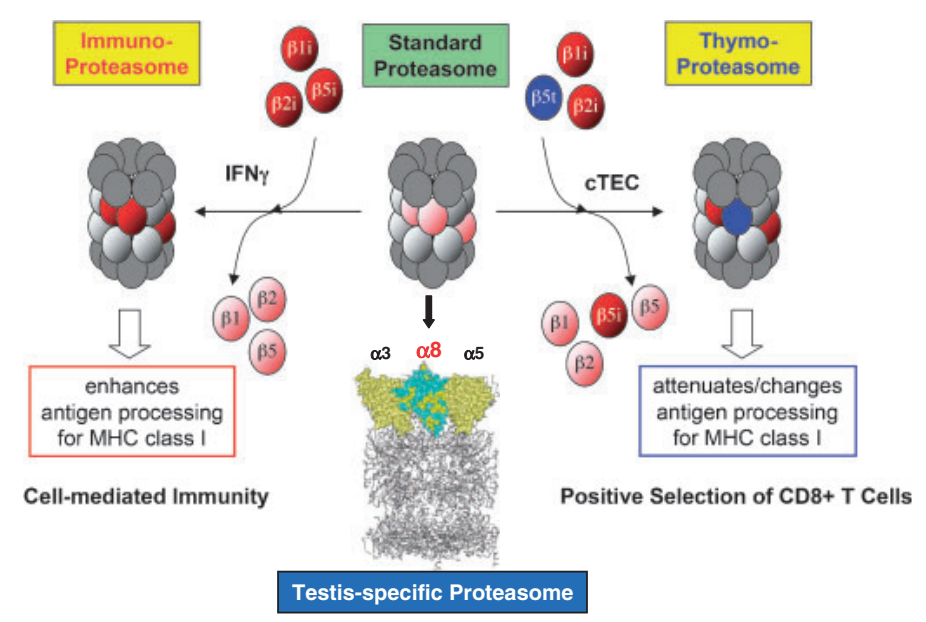

Fig. 3. Schematic representation of diverged proteasomes. 20S proteasomes are responsible for the proteolytic activity of the proteasomes and are composed of 28 subunits arranged as a cylinder containing four heteroheptameric rings with an $\alpha_{1-7} \beta_{1-7} \beta_{1-7} \alpha_{1-7}$ arrangement (middle-upper drawing, constitutive or standard proteasomes). In vertebrates, the $\beta 1 \mathrm{i}, \beta 2 \mathrm{i}$ and $\beta 5 \mathrm{i}$ subunits are expressed in response to IFN- $\gamma$ and are preferentially incorporated into proteasomes, resulting in the immunoproteasomes (left). The newly identified $\beta 5 \mathrm{t}$ catalytic subunit of $20 \mathrm{~S}$ proteasomes is incorporated in place of $\beta 5$ or $\beta 5 \mathrm{i}$, together with $\beta 1 \mathrm{i}$ and $\beta 2 \mathrm{i}$ to form thymoproteasomes, which are specifically found in cTECs (right). The middle-lower drawing represents the putative testis-specific proteasome, in which $\alpha 4$ of mammalian standard proteasomes is replaced by a novel subunit, $\alpha 8$.

Intriguingly, PR39, a 39-amino acid cell-permeable antibiotic peptide composed of $49 \%$ proline and $24 \%$ arginine is a noncompetitive and reversible inhibitor of $20 \mathrm{~S}$ proteasomes. ${ }^{77)}$ PR39 changes the conformational dynamics of the proteasome following their interaction, inducing a unique allosteric process that allows specific inhibition of degradation of selected proteins. ${ }^{78)}$ The suppressive effect of PR39 is universal for proteasomes from yeast to human, although its physiological role remains unknown.

\section{Diversity of Proteasomes}

The proteasome complex has been highly conserved during evolution due to its fundamental roles in cells. Budding yeast has seven $\alpha$ - and $\beta$-type subunits, consistent with the seven-subunit $\alpha$ - and $\beta$-ring observed in the $20 \mathrm{~S}$ proteasome. In contrast, vertebrates have remarkably more than seven $\beta$ type subunits. Accordingly, the proteasome system in vertebrates has acquired considerable diversity among the catalytic subunits, which have evolved during the acquisition of adaptive immunity. In this section, I describe the diverse functions of the proteasome with particular emphasis on its immunological roles. Moreover, flies and plants contain multiple proteasome genes (e.g., duplicated or triplicated), though their biological roles are unknown. ${ }^{79), 80)}$ For example, the Arabidopsis thaliana encodes $13 \alpha$-subunits and $10 \beta$-subunits of $20 \mathrm{~S}$ proteasomes. ${ }^{81)}$ In this section, I summarize advances related to this issue.

7.1 Immunoproteasome. Which are structurally related to $\beta 1, \beta 2$ and $\beta 5$, respectively (Table 2). The expression of these three subunits with highly similar amino acid sequences to other $\beta$ subunits indicates that IFN- $\gamma$ may cause the $\beta 1, \beta 2$ and $\beta 5$ subunits to be replaced with $\beta 1 \mathrm{i}, \beta 2 \mathrm{i}$ and $\beta 5 \mathrm{i}$, respectively. Accordingly, we have proposed that IFN- $\gamma$-inducible proteasomes should be called 'immunoproteasomes' (Fig. 3) to emphasize their specialized functions in immune responses and to distinguish them from complexes containing only constitutively expressed subunits. ${ }^{50)}$ On the other hand, the $20 \mathrm{~S}$ proteasomes that include the constitutively expressed $\beta 1, \beta 2$ and $\beta 5$ catalytic subunits are often called standard or constitutive proteasomes. MHC class I molecules bind peptides produced through proteolysis of cytosolic proteins and display them on the cell surface. This mechanism enables CTLs to detect and destroy abnormal cells that contain viral or other foreign proteins or tumor antigens. More than 15 years ago, the proteasome was identified as a candidate for the 
enzyme that processes intracellular (or endogenous) antigens. To date, the roles of the immunoproteasome, which contributes to the efficient production of peptide epitopes for CTLs, have been highlighted in the MHC class I-restricted antigen-processing pathway and cell-mediated immunity. ${ }^{50)-52)}$

The three IFN- $\gamma$-regulated immunosubunits contain active threonine residues, indicating that the exchange of subunits induced by IFN- $\gamma$ is likely to confer different functions to the proteasome. In fact, IFN- $\gamma$ alters the proteolytic specificities of proteasomes, increasing their chymotrypsin- and trypsin-like activities to cleave peptide bonds on the carboxyl side of hydrophobic and basic aminoacid residues, respectively, and decreasing their caspase-like activities for peptides containing acidic amino acid residues. ${ }^{50), 52)}$ Comparison of the tertiary structures of the standard proteasome and the immunoproteasome using computer-assisted modeling suggested that the caspase-like activity is reduced and the chymotryptic activity is enhanced in the immunoproteasome. ${ }^{13)}$ The altered peptidase activities suggest that immunoproteasomes in IFN$\gamma$-treated cells should generate more peptides with hydrophobic or basic carboxyl termini and fewer peptides with acidic carboxyl termini. The peptides generated by the immunoproteasome are more likely to bind in the peptide-binding pocket of MHC class I molecules, because hydrophobic or basic (to a lesser extent) carboxyl terminal peptide residues normally serve as anchors for binding in these class I complexes. Thus, IFN- $\gamma$ promotes the production of immunoproteasomes with an altered proteolytic specificity that may be more appropriate for the immunological processing of antigens. ${ }^{50)-52)}$ It is likely that the acquisition of the immunoproteasomes during evolution enabled organisms to produce MHC class I ligands and combat pathogens more efficiently. Indeed, mice lacking $\beta 1 \mathrm{i}, \beta 2 \mathrm{i}$ or $\beta 5 \mathrm{i}$ display defective antigen processing and consequently compromised immune responses. ${ }^{82)-84)}$

Sequence comparison of $\beta$-type subunits of standard and immunoproteasome genes indicates that each subunit pair that is exchanged in response to IFN- $\gamma$ is encoded by genes derived from a common ancestor gene. ${ }^{50)} \mathrm{We}$ previously proposed a chromosomal duplication model to explain the emergence of the IFN- $\gamma$-regulated $\beta$-type subunits. ${ }^{85)}$ The basic assumption of this model is that all of the IFN- $\gamma$-regulated $\beta$-type immunosubunits emerged simultaneously as a result of duplication of the MHC region during a genome-wide duplication. ${ }^{50)}$

7.2 Thymoproteasome. Recently, we identified a novel catalytic subunit designated $\beta 5 \mathrm{t}$ that is expressed exclusively in cortical thymic epithelial cells (cTECs); these cells are responsible for positive selection of developing thymocytes. The mechanism underlying the thymus-specific expression of $\beta 5 \mathrm{t}$ is not known. $\beta 5 \mathrm{t}$ is structurally related to $\beta 5$ and $\beta 5 \mathrm{i}$ and is encoded by an intronless gene, in contrast to the three-exon $\beta 5$ and $\beta 5 \mathrm{i}$ genes. Whereas the chymotrypsin-like activity of proteasomes is thought to be important for production of peptides with high affinities for MHC class I clefts, incorporation of $\beta 5 \mathrm{t}$ into proteasomes instead of $\beta 5$ or $\beta 5 \mathrm{i}$ selectively reduces this activity. Although this characteristic differs from the imunoproteasome, the high overall sequence similarity suggests these subunits belong to the same family. $\beta 5 \mathrm{t}$ and the immunosubunits $\beta 1 \mathrm{i}$ and $\beta 2 \mathrm{i}$, but not their standard counterparts, are incorporated into a vertebrate-specific alternative $20 \mathrm{~S}$ proteasome referred to as the 'thymoproteasome' (Fig. 3). ${ }^{86)} \mathrm{We}$ found that $\beta 5$ t-deficient mice displayed major (but not total) defects in the thymic development of CD ${ }^{+} \mathrm{T}$ cells, although no obvious abnormality was observed in the thymic architecture, suggesting that $\beta 5 \mathrm{t}$ is not essential for the differentiation and proliferation of cTECs. Importantly, no obvious alterations in the $\mathrm{CD} 4^{+} \mathrm{CD} 8^{+}$(co-receptors of TCR) double-positive thymocytes and CD $4^{+} \mathrm{T}$ cell populations were observed in $\beta 5 \mathrm{t}^{-/-}$mice. These results suggest a key role for $\beta 5 \mathrm{t}$ in the development of the $\mathrm{MHC}$ class I-restricted $\mathrm{CD} 8^{+} \mathrm{T}$ cell repertoire during thymic selection.

During positive selection, double-positive cells that interact with self-peptide-MHC complexes expressed on cTECs with a sufficiently modest avidity [i.e., affinity (MHC-TCR interaction) X density (surface MHC levels)] are rescued from intrathymic death and induced to differentiate into $\mathrm{CD}^{+}{ }^{+}$or $\mathrm{CD}^{+}$single-positive thymocytes. In contrast, double-positive cells that interact with high avidity with self-peptide-MHC complexes are eliminated through apoptosis, a process referred to as negative selection. ${ }^{87-89)}$ In addition, thymocytes that lack functional $\mathrm{T}$ cell receptors also undergo apoptosis, a process referred to as null selection. To 
date, however, the mechanism by which cTECs provide the specialized signals for positive selection has not been elucidated. Considering that proteasomes are essential for the production of MHC class I ligands and that $\beta 5 \mathrm{t}$ specifically attenuates the proteasomal chymotryptic activity without changing the caspase- and trypsin-like activities, it is possible that thymoproteasomes in cTECs predominantly produce moderate avidity MHC class I ligands rather than high-affinity ligands, which would support positive selection. The discovery of the thymoproteasome may contribute to our understanding of how positive selection occurs in the thymus. The types of antigenic peptides generated by the thymoproteasome, the underlying mechanism, and the roles in positive selection require further examination.

Like the immunoproteasome genes, the $\beta 5 \mathrm{t}$ and PA28 $\alpha / \beta$ genes appear to result from modification and duplication of existing nonimmune genes, such as $\beta 5$ and PA28 $\gamma$, respectively, and may have been instrumental in the emergence of the adaptive immune system.

7.3 Other subtypes of proteasomes. Surprisingly, in Drosophila, about a third of the 32 proteasome subunits have testis-specific isoforms, encoded by paralogous genes. ${ }^{90)}$ Analysis of GFPtagged transgenes showed that whereas the Drosophila $\alpha 6$ (Pros $\alpha 6)$ subunit is expressed in early stages of spermatogenesis, gradually fading away following meiosis, the expression of testis-specific Pros $\alpha 6 \mathrm{~T}$ becomes prominent in spermatid nuclei and cytoplasm after meiosis, and persists in mature sperms. Moreover, a loss-of-function mutant of Pros $\alpha 6$ T reveals that homozygous males are sterile and show spermatogenic defects in sperm individualization and nuclear maturation, consistent with the expression pattern of Pros $\alpha 6 \mathrm{~T}$, indicating a functional role for testis-specific proteasomes during Drosophila spermatogenesis. ${ }^{90)}$

Interestingly, although examination of the various $\alpha$-rings shows that most organisms, except flies and plants have seven $\alpha$ subunits, an eighth $\alpha$ subunit (PAMA8: 20q13.33) that resembles the ubiquitously expressed $\alpha 4$ subunit is present in humans (PAMA7: 11q11.2; our unpublished results). The limited expression of the $\alpha 8$ subunit in cells and tissues, mainly in the testis, implies that it is involved in spatially and temporally restricted regulatory programs. Here I propose the term 'mammalian testis-specific proteasome' for the proteasome complex containing the $\alpha 8$ subunit instead of $\alpha 4$ (see Fig. 3).

As described in a previous section, Rpn10 functions as a receptor to trap polyubiquitylated client proteins for ultimate breakdown by the $26 \mathrm{~S}$ proteasome. Intriguingly, mouse Rpn10 mRNA occurs in at least five distinct forms, Rpn10a-e, due to developmentally regulated alternative splicing. ${ }^{91)}$ These isoforms, with the exception of the universally expressed Rpn10a, are expressed in tissue-specific and/or developmental stage-specific manners. For example, Rpn10e is specifically expressed in the embryonic brain in mice, implying the existence of 'the brain-specific proteasome'. Knocking out the mouse Rpn10 gene was embryonically lethal, ${ }^{92)}$ although the specific reason was not determined. It is interesting that Rpn10a knock-in mice lacking the Rpn10 gene are born without any apparent abnormalities, suggesting that Rpn10a is a particularly important Rpn10 family protein.

Intriguingly, protein misfolding caused by exposure to arsenite induces the expression of arsenite-inducible proteasomal 19S regulatory-associated protein (AIRAP), which binds to Rpn1. Cells lacking AIRAP contain more polyubiquitylated proteins and exhibit higher levels of stress when exposed to arsenite, suggesting that AIRAP adapts the core protein degradation machinery to counteract the proteotoxicity of this environmental toxin. ${ }^{93}$ In mammals, a second constitutively expressed AIRAP-like gene (AIRAPL) encodes a proteasome-interacting protein. ${ }^{94)}$ Whereas most AIRAP is associated with the $26 \mathrm{~S}$ proteasome, AIRAPL is detected as a free form; exposure to arsenite, however, shifts some of this protein to heavier fractions that also contain the AIRAP peak colocalized with the $26 \mathrm{~S}$ proteasome. Therefore, proteasomes containing these newly described subunits may be referred to as 'stress-specific proteasome'.

\section{Proteasome Assembly}

How the complex structures of the $20 \mathrm{~S}$ and $26 \mathrm{~S}$ proteasomes are organized remains largely unknown. For example, the mechanism responsible for the correct positioning of the various sets of different, but structurally-related subunits in the $20 \mathrm{~S}$ proteasome is unclear. Recent studies have 
examined this issue, particularly the biogenesis of eukaryotic 20S proteasomes from 28 subunits, each of which occupies a defined position within the $20 \mathrm{~S}$ proteasome particle.

8.1 Assembly of $20 \mathrm{~S}$ proteasome. The prokaryotic $20 \mathrm{~S}$ proteasome consists of homo-oligomers of the same $\alpha$ and $\beta$ subunits; these subunits can assemble autonomously into functionally mature proteasomes without the help of any chaperone proteins. ${ }^{11)}$ On the other hand, the assembly of the eukaryotic 20S proteasome is more complex; it requires a set of extrinsic [i.e., proteasome assembling chaperones (PAC) 1-4 and Ump1] and intrinsic [i.e., propeptides and C-tail of $\beta$ subunits] chaperones (Table 2). ${ }^{95)-99)}$ Among these potential regulatory processes, current studies aim to clarify the assembly mechanism of $20 \mathrm{~S}$ proteasomes in yeast and human cells, which share common assembly and intramolecular chaperones, although some of their roles differ considerably. ${ }^{98)}$

8.1.1 $\alpha$-ring formation assisted by extrinsic chaperones. The first assembly step for the eukaryotic $20 \mathrm{~S}$ proteasome is $\alpha$-ring formation, which was thought to occur autonomously before the identification of multiple chaperone molecules specialized for proteasome assembly. Remarkably, recent studies identified two heterodimeric complexes dedicated to proteasome assembly in human cells (PAC1-PAC2 and PAC3-PAC4) and in yeast (Pba1/Poc1, Pba2/Poc2, Pba3/Poc3/Dmp2, and Pba4/Poc4/Dmp1; orthologs of human PAC1, PAC2, PAC3 and PAC4, respectively) (Fig. 4). ${ }^{98)}$

Knockdown of PAC1 expression using siRNA resulted in the loss of $\mathrm{PAC} 2$, and vice versa, indicating that PAC1 and PAC2 are stable only when they form a heterodimer. ${ }^{100)}$ Coexpression of PAC1 and PAC2 in Escherichia coli supported this hypothesis. The human PAC1-PAC2 heterodimer promotes $\alpha$-ring formation in vitro and is mainly associated with an $\alpha$-subunit proteasome assembly intermediate in vivo. Intriguingly, knockdown of PAC1 or PAC2 expression decreased the number of $\alpha$-rings and resulted in accumulation of off-pathway products, presumably unusual $\alpha$-ring dimers. These findings indicate that PAC1-PAC2 does not only promote $\alpha$-ring formation but also prevents aberrant dimerization of the $\alpha$-rings, which seems to be an intrinsic characteristic of $\alpha$-rings. PAC1-PAC2 binds to proteasome precursors until the complete formation of the $20 \mathrm{~S}$ proteasome; the complex is

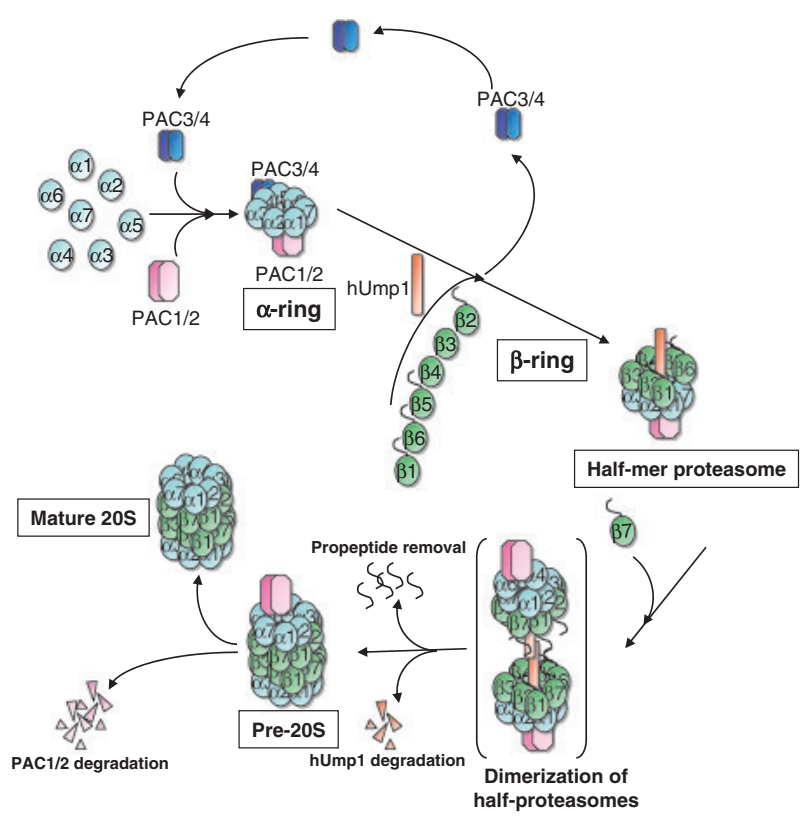

Fig. 4. A schematic model of mammalian 20S proteasome assembly. The PAC1-PAC2 and PAC3-PAC4 heterodimeric complexes are involved in the formation of the $\alpha$-ring. Then, sequential incorporation of the $\beta$ subunits begins with the binding of $\beta 2$ and hUmp1 on the $\alpha$-ring. hUmp1 is required for the association of $\beta 2$ in early assembly intermediates. PAC3PAC4, which is released at the time of $\beta 3$ association, maintains the structural integrity of the intermediates until $\beta 3$ is incorporated on the $\alpha$-ring. The subsequent ordered incorporation of other $\beta$ subunits is assisted by intramolecular chaperones, such as the propeptides of $\beta 2$ and $\beta 5$ and the $\mathrm{C}$ terminal tail of $\beta 2$. Dimerization of half-mers (i.e., halfproteasomes lacking $\beta 7$ ) is assisted by the C-terminal tail of $\beta 7$. This is followed by removal of the $\beta$ subunit propeptides $(\beta 1, \beta 2, \beta 5, \beta 6$ and $\beta 7)$ as well as hUmp1 and PAC1-PAC2 degradation. Note that the role of Ump1 for dimerization of half-proteasomes or checkpoint of half-mers is emphasized in yeast, but its exact role is somewhat difference in mammals (for details, see text).

ultimately degraded by the newly formed $20 \mathrm{~S}$ proteasome and therefore has a half-life of approximately 30 minutes, which agrees with the maturation period of the $20 \mathrm{~S}$ proteasome. In budding yeast, Pba1-Pba2 appears to be the counterpart of human PAC1-PAC2 because Pba1 and Pba2 have similar sequences to those of PAC1 and PAC2, respectively, though the degree of sequence identity is marginal. Although the yeast and human proteins share several characteristics, such as heterodimer formation, association with the $\alpha$-ring assembly intermediate, and short half-life, yeast strains lacking Pba1-Pba2 displayed only subtle de- 
fects. ${ }^{101)}$ This contrasts with mammals, because PAC1 deficiency in mice caused early embryonic lethality (see below).

PAC3 and PAC4 were copurified with $\alpha$-rings and PAC1-PAC2 in human cells (Ref. 102 and unpublished results). Knockdown of PAC3 and PAC1 expression produced additive effects on proteasome assembly; simultaneous depletion of PAC1 and PAC3 caused severe reduction in cellular levels of $\alpha$-rings and 20S proteasomes compared with depletion of either PAC1 or PAC3 alone. These findings suggest that $\mathrm{PAC} 1-\mathrm{PAC} 2$ and PAC3-PAC4 function differently but cooperate with each other in the assembly of $\alpha$-rings and half-proteasomes.

Pba3, the ortholog of PAC3 in budding yeast, and its binding partner $\mathrm{Pba} 4$, were identified by several groups independently. ${ }^{103)-106)} \mathrm{Pba3}$ and $\mathrm{Pba} 4$ form a heterodimer complex, similar to PAC3 and PAC4; this complex specifically binds to proteasome precursors containing all seven $\alpha$ subunits and the unprocessed $\beta 2$ subunit in vivo, whereas $\mathrm{Pba} 3-\mathrm{Pba} 4$ was found to interact directly and specifically with $\alpha 5$ in vitro. ${ }^{104)}$ Deletion of $\mathrm{Pba} 3$ or Pba4 in cells markedly decreased the level of $20 \mathrm{~S}$ proteasomes and caused accumulation of assembly intermediates. ${ }^{105)}$ Taken together, these results suggest that $\mathrm{Pba} 3-\mathrm{Pba} 4$ catalyzes correct subunit orientation in the $\alpha$-ring, presumably by collaborating with PAC1-PAC2 and facilitating the recruitment of $\alpha$ subunits.

X-ray crystallography revealed that the quaternary structure of the $\mathrm{Pba} 3-\mathrm{Pba} 4$ heterodimer displays profound similarity to that of the PAC3 homodimer, which may not exist in vivo, despite the divergence of the primary structures of these subunits. ${ }^{104)}$ The tertiary structures of all of the $\alpha$ and $\beta$ subunits closely resemble each other, which, together with the obvious homology in their sequences, imply that they were derived from a common ancestral gene. Interestingly, the overall structure of the $\mathrm{Pba} 3-\mathrm{Pba} 4$ complex also resembles those of proteasomal $\alpha$ - and $\beta$-subunits. Structural analysis of the $\mathrm{Pba} 3-\mathrm{Pba} 4-\alpha 5$ complex revealed that $\mathrm{Pba} 3-\mathrm{Pba} 4$ binds on the surface of the $\alpha$-ring at a position where the $\beta$ subunits are assembled. The binding mode of Pba3-Pba4, however, is different from that of the $\beta$ subunits; Pba3-Pba4 is located near the inner surface of the $\alpha$-ring, which enables this complex to interact with three different $\alpha$ subunits, $\alpha 4, \alpha 5$ and $\alpha 6$, whereas the $\beta$ subunits interact with two neighboring $\alpha$ subunits. This feature of $\mathrm{Pba} 3-\mathrm{Pba} 4$ might be helpful for initiating $\alpha$-ring assembly. The location of Pba3-Pba4 is consistent with the results of biochemical analyses, which indicate that Pba3-Pba4 and PAC3-PAC4 detach from the $\alpha$-rings during $\beta$-ring formation. Unfortunately, the molecular roles of PAC1-PAC2 in the assembly process are unclear, because structural information about PAC1-PAC2 and Pba1-Pba2 are not yet available. However, one can predict that PAC1-PAC2 makes contact with the surface of the $\alpha$-ring, because this chaperone complex prevents the spontaneous dimerization of $\alpha$-rings (i.e., the formation of off-pathway assemblies). On the other hand, Ump1 may posit inside in the newly formed proteasome, because it can associate with $\alpha$-ring and is protected by tryptic digestion in vitro. Based on the findings that none of these dedicated chaperones is strictly dispensable in the budding yeast, and together with the observations that the $\alpha$ - and $\beta$-subunits can self-assemble in vitro, forming off-pathway products, unassisted assembly may be error prone. ${ }^{97)}$ However, genetic ablation of mouse orthologs of Ump1 or PAC1 caused early embryonic lethality (our unpublished observations), emphasizing the importance of the chaperone-mediated proteasome assembly pathway in embryonic development.

8.1.2 $\beta$-ring formation assisted by intramolecular chaperones. No $\beta$-ring assembly intermediates have been detected in cells, implying that the halfproteasome is not formed through the association of an $\alpha$-ring with a $\beta$-ring. Rather the $\alpha$-ring serves as a scaffold for the assembly of the $\beta$ subunits; i.e., each $\beta$ subunit is progressively added to the complex on the $\alpha$-ring, resulting in half-proteasomes that consist of one copy each of the $\alpha$ - and $\beta$ rings. siRNA-mediated silencing of the expression of each $\beta$ subunit in mammalian cells caused the accumulation of an "assembly-arrested" intermediate, representing the structure just before incorporation of the knocked-down $\beta$ subunit. This technique allowed the order of $\beta$-subunit assembly on the $\alpha$-ring to be defined: $\beta 2$, followed by $\beta 3, \beta 4, \beta 5$, $\beta 6, \beta 1$ and $\beta 7$ (Fig. 4). Although $\beta 1$ has been experimentally incorporated at various steps, its incorporation most likely follows that of $\beta 6$. A recent report using yeast showed that the addition of other $\beta$ subunits, excluding $\beta 7$, form another 
intermediate referred to as the "half-mer" precursor complex. ${ }^{101)}$ During $\beta$-ring assembly in human cells, release of PAC3 (and perhaps with PAC4) is coupled to $\beta 3$ incorporation, which is consistent with the observation that $\mathrm{Pba} 3-\mathrm{Pba} 4$ was selectively copurified with $\beta 2$ but not with other $\beta$ subunits in yeast, indicating a conserved mechanism in the roles of PAC3-PAC4 and Pba3-Pba4 during proteasome assembly.

Propeptides and the tails of $20 \mathrm{~S}$ proteasome $\beta$ subunits facilitate proteasome assembly; these types of domains are called 'intramolecular chaperones' ${ }^{107)}$ The N-terminal propeptides and C-terminal tails of $\beta$ subunits play pivotal roles in proteasome assembly through specific interactions with cis and trans $\beta$-rings in yeast and humans. For example, the propeptide from $\beta 2$ influences cooperative proteasome assembly. ${ }^{108)}$ The $\beta 5$ propeptide facilitates the incorporation of this subunit and is essential for yeast viability. ${ }^{109)}$ On the other hand, the $\beta 5$ propeptide does not appear to be required for incorporation of $\beta 5$ but rather it is used for $\beta 6$ recruitment in human cells. ${ }^{110)}$ The propeptides of $\beta 1$ and $\beta 2$ are dispensable for cell viability in yeast, although mutants lacking these two propeptides displayed subtle defects in proteasome biogenesis. Thus, the role(s) of these propeptides remains obscure. In human cells, loss of the $\beta 2$ propeptide eliminated $\beta 3$ recruitment and was thus fatal to the cells. Of note, the C-terminal tail of $\beta 2$, which wraps around $\beta 3$ within the same $\beta$-ring, is also essential for proteasome biogenesis both in yeast and human cells.

Interestingly, the amino-acid sequences of the human $\beta$-subunit propeptides are considerably different from those of their yeast counterparts, unlike the mature $\beta$ subunits, which are well conserved between yeast and humans. Such differences are also found in the extrinsic proteasome assembly chaperones, such as PAC1-4 and Ump1 (i.e., 5-20\% identity), as discussed previously. ${ }^{98)}$ Why the chaperones have diverged during evolution is unknown; nonetheless, their basic functions and tertiary structures are highly conserved.

Unexpectedly, intermediates resulting from siRNA-mediated knockdown of each $\beta$ subunit accumulated as two major and minor bands, in which the composition of each major and minor band in terms of $\alpha$ and $\beta$ subunits was identical. ${ }^{110)}$ PA28 was associated with the slow-migrating minor bands, different from PAC1 and $\mathrm{Hsp} 90 \alpha$, which were detected only in the major bands. Hsc70 was observed in both the major and minor bands. Neither Hsp90 $\alpha$ nor Hsc70 was detected in the $\alpha$ ring. At present, it is unknown whether these conventional chaperones really have any roles in proteasome biogenesis or whether they are merely associated with the intermediates as experimental artifacts.

8.1.3 Role of another chaperone Ump1 and dimerization of half-proteasomes. Ump1 was identified in mutant yeast defective for $\underline{u}$ biquitin-mediated proteolysis and is the first identified extrinsic assembly factor for $20 \mathrm{~S}$ proteasomes. ${ }^{111)} \mathrm{Ump} 1$ specifically associates with the assembly intermediates of $20 \mathrm{~S}$ proteasomes and appears to enter the assembly pathway after association of $\beta 2, \beta 3$ and $\beta 4$ in yeast. Upon dimerization of the half-proteasomes, Ump1 is encapsulated and degraded within the newly formed 20S proteasome like PAC1 and PAC2. Loss of Ump1 caused accumulation of assembly intermediates as well as half-proteasomes with unprocessed $\beta$ subunits, indicating that Ump1 coordinates the processing of $\beta$ subunits and dimerization of half-proteasomes in yeast. ${ }^{111)}$ On the other hand, Ump1 is also thought to function as an assembly checkpoint factor that inhibits dimerization of half-proteasomes until a full set of $\beta$ subunits have been recruited to the $\alpha$-ring. ${ }^{101)}$

The human ortholog of Ump1 (hUmp1, Proteassemblin, or POMP) was identified using homology searches. ${ }^{12), 113)}$ hUmp1 is included in precursor proteasomes with unprocessed $\beta$ subunits and is degraded upon completion of proteasome assembly with a similar half-life to that of PAC1-PAC2. ${ }^{110}$ ) Interestingly, knockdown of hUmp1 expression inhibited $\beta 5$ recruitment, and resulted in the accumulation of $\alpha$-rings with no $\beta$ subunits. Moreover, hUmp1 can bind to the $\alpha$-ring in the absence of $\beta$ subunits and incorporation of hUmp1 is coupled with $\beta 2$ binding, suggesting that hUmp1 is incorporated into proteasome precursors earlier than yeast Ump1. Therefore, hUmp1 is required for the initiation of $\beta$-ring formation, differing from the reported role of yeast Ump1. In the final step of $\beta$ ring assembly, the $\mathrm{C}$-terminal tail of $\beta 7$ is inserted into a groove between $\beta 1$ and $\beta 2$ in the opposite half-mer precursor, which triggers dimerization of the half-proteasomes in both yeast and humans. ${ }^{68), 101)}$ Correct dimerization of half-protea- 
somes is followed by removal of the $\beta$ propeptides and degradation of Ump1 and PAC1-PAC2 (for details, see Ref. 98.) (Fig. 4).

8.2 Assembly of immune response proteasomes. Vertebrates encode four additional catalytic $\beta$-subunits: IFN- $\gamma$-inducible $\beta 1 \mathrm{i}, \beta 2 \mathrm{i}$ and $\beta 5 \mathrm{i}$ and thymus-specific $\beta 5 \mathrm{t}$ (Fig. 3 ). These alternative proteasomes play key roles in acquired/ adaptive immunity by altering antigen processing as mentioned above. Accumulating evidence has clarified the molecular mechanism of immunoproteasome assembly. ${ }^{98)}$ Despite the coexistence of both immunoproteasome and standard subunits in some cells, immunoproteasomes are preferentially assembled. ${ }^{114)}$ The propeptides of the immunosubunits and hUmp1 play key roles in this cooperative assembly. ${ }^{115)}$ Interestingly, $\beta 1 \mathrm{i}$ enters the assembly pathway of immunoproteasomes earlier than in the standard proteasome assembly process, resulting in an assembly intermediate containing the $\alpha$-ring, $\beta 1 \mathrm{i}, \beta 2 \mathrm{i}, \beta 3$ and $\beta 4$. In this intermediate, incorporation of $\beta 2 \mathrm{i}$ depends on $\beta 1 \mathrm{i}$, and incorporation of $\beta 1 \mathrm{i}$ is facilitated by $\beta 2 \mathrm{i}$. $\beta 5 \mathrm{i}$ is incorporated preferentially over $\beta 5$ into the intermediates containing $\beta 1 \mathrm{i}$ and $\beta 2 \mathrm{i}^{114}$ ) This interdependency supports the homogenous formation of immunoproteasomes containing all three inducible subunits. Indeed, $\beta 2 \mathrm{i}$ processing and incorporation is severely impaired in $\beta 1 \mathrm{i}$-deficient cells, and $\beta 1 \mathrm{i}$ incorporation is partially inhibited in $\beta 2 \mathrm{i}$-deficient cells, whereas $\beta 5 \mathrm{i}$ incorporation, which is dependent on the $\beta 5 \mathrm{i}$ propeptide but not $\beta 5 \mathrm{i}$ catalytic activity, is not affected in either of these mutant cell lines. ${ }^{110)}$ $\beta 5$ i-deficient cells exhibited significantly retarded proteasome assembly and accumulation of proteasome precursors containing unprocessed $\beta 1 \mathrm{i}$ and $\beta 2$ i. Intriguingly, IFN- $\gamma$ stimulation increased transcription of hUmp1 and immunosubunit mRNA, but decreased hUmp1 protein levels due to $\sim 4$-fold augmentation of hUmp1 protein turnover. ${ }^{116)}$ This rapid turnover was coupled with the maturation of active immunoproteasomes, indicating that the rate of immunoproteasome generation is four times faster than that of standard proteasomes. The higher affinity of hUmp1 for $\beta 5 \mathrm{i}$ than for $\beta 5$ is likely to contribute to the rapid maturation of immunoproteasomes. ${ }^{116)}$

How the thymoproteasome, another vertebrate-specific $20 \mathrm{~S}$ proteasome, is assembled is currently unknown. When $\beta 5 \mathrm{t}$ was ectopically expressed in a human cell line that does not express immunosubunits, the protein was readily processed and incorporated into the proteasome, suggesting that $\beta 5 \mathrm{t}$ is preferentially incorporated compared with $\beta 5$ and that $\beta 1 \mathrm{i}$ and $\beta 2 \mathrm{i}$ (i.e., partners of thymoproteasomes) are not required for $\beta 5 \mathrm{t}$ incorporation. ${ }^{87)}$ Because the majority of proteasomes in cTECs are thymoproteasomes, it is thought that $\beta 5 \mathrm{t}$ is preferentially incorporated before $\beta 5 \mathrm{i}$ in the thymus, suggesting that thymoproteasomes employ a specific assembly mechanism. Indeed, considering the high expressions of $\beta 1 \mathrm{i}$ and $\beta 2 \mathrm{i}, \beta 5 \mathrm{i}$ whose gene and $\beta 2 \mathrm{i}$ gene are located at the same MHC class II region must be expressed in cTECs. According to the scenario for the immunoproteasome assembly, it is plausible that the propeptide or the extended Cterminal tail of $\beta 5 \mathrm{t}$ contributes to the assembly of the thymoproteasome as an intramolecular chaperone, but there is no available information at present in support of this assumption. ${ }^{98)}$

\subsection{Assembly of 19S RP and 26S pro-} teasome. Currently, the assembly mechanism for the 19S RP is poorly understood. The yeast lid complex seems to be subdivided into two clusters: one is made up of Rpn5, Rpn6, Rpn8, Rpn9 and Rpn11, and the other contains Rpn3, Rpn7, Rpn12 and Rpn15. The interaction between Rpn3 and Rpn5 connects these two clusters, implying a hierarchy in the incorporation of Rpn subunits into the lid complex. ${ }^{117)}$ Recently, it was proposed that the 20S proteasome functions as an assembly factor for the RP due to aberrant RP formation in the presence of defective $20 \mathrm{~S}$ proteasomes in yeast. ${ }^{105)}$ It was also proposed that the base and the lid are assembled independently, and then joined together. ${ }^{118}$ ) The base is composed of six related AAA-ATPase subunits and four non-ATPase subunits. Putative chaperones may discriminate and arrange the six homologous ATPase subunits in a defined order, as is observed in the assembly of $20 \mathrm{~S} \alpha$-ring. Whether assembly chaperones are required for the assembly of the ATPase ring, the lid, the base, and/or the 19S RP complex requires further studies.

The assembly mechanism of the $26 \mathrm{~S}$ proteasome is largely not understood. Hsp90 is thought to play a role in both the assembly and maintenance of the lid in yeast. ${ }^{119)}$ Inactivation of Hsp90 was found to cause disassembly of the lid complex, which was then partially reassembled into the $26 \mathrm{~S}$ proteasome 
following reactivation of Hsp90 in vivo or by adding Hsp90 and ATP in vitro. These findings suggest that the ATP-dependent chaperone activity of Hsp90 contributes to the assembly of the lid and $26 \mathrm{~S}$ proteasomes. The function of Hsp90 in the assembly of $26 \mathrm{~S}$ proteasomes, however, remains to be elucidated. Inhibition of proteasome active sites also stabilized $26 \mathrm{~S}$ proteasomes, suggesting that the interface between the RP and the 20S proteasome changes depending on the activities of the $20 \mathrm{~S}$ proteasome. ${ }^{120)}$ Related to this result, whether $26 \mathrm{~S}$ proteasomes undergo obligatory disassembly and reassembly during protein degradation is currently a point of debate in this field. It was first reported that disassembly of the $26 \mathrm{~S}$ proteasome and dissociation of the RP into subcomplexes or subunits are induced upon ATP-dependent degradation of a substrate protein in yeast. ${ }^{121)}$ In contrast, it was more recently reported that mammalian $26 \mathrm{~S}$ proteasomes can degrade polyubiquitylated proteins without disassembling or the release of any subunits or subcomplexes. ${ }^{122)}$

\section{Proteasome Interacting Proteins (PIPs)}

Recent proteomic analyses have identified auxiliary factors with known and unknown functions that are physically and/or transiently associated with the $26 \mathrm{~S}$ proteasome. ${ }^{123)-125)}$ These proteins, referred to as proteasome-interacting proteins (PIPs), can be categorized into two groups (Table 1). The first group contains protein factors that are related to the ubiquitylation system. In this article, I described the association of the deubiquitylating enzymes Usp14 and Uch37 with the base subunits Rpn1 and Rpn2 via Rpn13, respectively. The extrinsic UBL-UBA ubiquitin receptors may also belong to this group. In addition, emerging evidence indicates that many ubiquitin E3 ligases, such as Hul5/KIAA10, E6AP, and Parkin, are transiently associated with the $26 \mathrm{~S}$ proteasome. Moreover, other E3s such as Ubr1, $\mathrm{APC}, \mathrm{Ufd} 4$ and $\mathrm{SCF}^{\mathrm{CDC} 4}$ as well as some E2 enzymes are also reported to associate loosely with the $19 \mathrm{~S}$ RP of $26 \mathrm{~S}$ proteasomes. ${ }^{9)}$

The second group contains auxiliary factors that regulate proteasome functions via direct binding. For example, Ecm29 is an approximately 200$\mathrm{kDa}$ protein that can bind to both the RP and the $20 \mathrm{~S}$ proteasome in yeast. Purified 26S proteasomes from $\Delta e c m 29$ cells tend to dissociate into RPs and
$20 \mathrm{~S}$ proteasomes. Together with the findings of electron micrographs of Ecm29-20S proteasome complexes, these results suggest that Ecm29 stabilizes the $26 \mathrm{~S}$ proteasomes by tethering the $20 \mathrm{~S}$ proteasome to the RP. ${ }^{126), 127)}$ The mechanism underlying this function, however, is unclear. As listed in Table 2, there are many other factors, such as p28/gankyrin, Rpn14, p27 and S5b that interact with proteasomes. Some of them are suggested to be responsible for the regulation of $26 \mathrm{~S}$ proteasomes or the assembly of the lid and base complexes, the process is largely ambiguous to date, but the details of their functions are unknown and require further studies.

\section{Perspectives}

The UPS is essential for cells to proliferate, and consequently proteasome levels are tightly regulated. For example, the balance between 20S and 26S proteasomes fluctuates to respond to environmental conditions; e.g., while the $26 \mathrm{~S}$ proteasome levels increase during growth and developmental stages the $26 \mathrm{~S}$ proteasome attenuates with aging process in Drosophila. ${ }^{128)}$ In addition, proteasomes are predominantly distributed in the nuclei of rapidly proliferating mammalian cells and growing yeast, indicating that this localization may contribute to cell proliferation. Why the proteasome is predominantly located in this cellular compartment remains to be determined, although typical nuclear localization signals (NLSs) are found on several of the 20S proteasomal $\alpha$ subunits, but not the $\beta$ subunits. ${ }^{129)}$ No clear NLSs have been identified in the 19S RP subunits, except Rpn2, but it is plausible that the lid and the base are transported into the nucleus independently (unpublished results); the mechanisms underlying this translocation are a complete mystery at present. In addition, the issue of nuclear export (i.e., nucleocytoplasmic transport) of proteasomes is totally open to investigation. Indeed, nuclear export signals (NESs) of $20 \mathrm{~S}$ and $26 \mathrm{~S}$ proteasomes remain undefined.

To date, various lines of evidence have supported the importance of proteasomes outside of their proteolytic functions, such as transcription, DNA repair, and chromatin modeling. 9) For example, the 19S RP may contribute to transcriptional control in cells, independent of the functions of the 20S proteasome. ${ }^{130), 131)}$ The non-proteolytic activities of the proteasome are important for co- 
activator recruitment; i.e., the ATPase activity of PA700 drives a stable association of a transactivator with the SAGA histone acetyltransferase complex. ${ }^{131)}$ PA700 also acts nonproteolytically in nuclear excision repair (NER). ${ }^{132), 133)}$ Chromatin remodeling is another nonproteolytic role of PA700, with implications for both transcription and DNA repair. ${ }^{131)}$ In addition, a proteasome-derived ATPase activity mediates relocalization of the substrates of endoplasmic reticulum-associated degradation (ERAD), a function that is primarily attributed to the AAA-ATPase p97/Cdc48. ${ }^{134)}$ ERAD eliminates aberrant proteins from the ER by localizing them to the cytoplasm where they are tagged by ubiquitin and degraded by the proteasome.

As described before, PI31 and PR39 are naturally occurring proteasome inhibitors, but their physiological functions are unclear. On the other hand, membrane-permeable synthetic inhibitors have been devised; e.g., various substrate-related peptidyl aldehydes have been designed as potent inhibitors of proteasomes, such as MG-132 ( $N$ carbobenzoxy-Leu-Leu-leucinal) and PSI ( $N$-carbobenzoxy-L-gamma-t-butyl-L-glutamyl-L-alanyl-Lleucinal), and the non-aldehyde peptidyl inhibitor Z$\mathrm{L}_{3} \mathrm{VS}$ (carboxybenzyl-leucyl-leucyl-leucine vinyl sulfone), which are often used in in vitro and in vivo experiments. ${ }^{135), 136)}$ However, caution must be exercised in their use for inferring proteasome functions, because they inhibit not only proteasomes but also cysteine proteases such as calpains and lysosomal cathepsins. ${ }^{135)}$ In contrast to these compounds, microbial metabolites, lactacystin and epoxomicin, were found to be selective proteasome inhibitors that do not affect other proteases examined so far. ${ }^{137), 138)}$ Of particular interest is bortezomib (also known as velcade or PS-341). Bortezomib as first-in-class proteasome inhibitor has proven to be highly effective in some hematological malignancies, and in fact it has been granted approval by the FDA for relapsed multiple myeloma and non-Hodgkin lymphoma (NHL) and has been used clinically in over 85 countries worldwide so far. ${ }^{139)}$ Moreover, preclinical studies demonstrate that proteasome inhibition potentiates the activity of other cancer therapeutics, and particularly, the combination of proteasome inhibition with novel targeted therapies is an emerging field in oncology. ${ }^{140)}$ Furthermore, Salinosporamide A (also called NPI-0052), ${ }^{141)}$ recently identified from the marine bacterium Salinispora tropica, is a potent inhibitor of $20 \mathrm{~S}$ proteasome and exhibits therapeutic potential against a wide variety of tumors. In addition, many other proteasome inhibitors are being assessed clinically for therapeutic use. ${ }^{142)}$ Thus, proteasome inhibitors provide a powerful new tool as fashionable drugs against cancer and other diseases including inflammations.

Finally, it should be emphasized that studies of the proteasome continue to provide significant insights in the physiologic roles of these complexes. Many questions, however, remain to be uncovered.

\section{Acknowledgements}

This work was supported by grants from the Ministry of Education, Culture, Sports, Science and Technology (MEXT) of Japan; the Target Protein Project of MEXT; and Health and Labor Science Research Grants.

\section{References}

1) Hershko, A. and Ciechanover, A. (1998) The ubiquitin system. Annu. Rev. Biochem. 67, 425-479.

2) Ravid, T. and Hochstrasser, M. (2008) Diversity of degradation signals in the ubiquitin-proteasome system. Nat. Rev. Mol. Cell Biol. 9, 679690.

3) Ventii, K. H. and Wilkinson, K. D. (2008) Protein partners of deubiquitinating enzymes. Biochem. J. 414, 161-175.

4) Varshavsky, A. (2005) Regulated protein degradation. Trends Biochem. Sci. 30, 283-286.

5) Ciechanover, A. (2006) The ubiquitin proteolytic system: from a vague idea, through basic mechanisms, and onto human diseases and drug targeting. Neurology 66, S7-19.

6) Tai, H. C. and Schuman, E. M. (2008) Ubiquitin, the proteasome and protein degradation in neuronal function and dysfunction. Nat. Rev. Neurosci. 9, 826-838.

7) Coux, O., Tanaka, K. and Goldberg, A. L. (1996) Structure and functions of the $20 \mathrm{~S}$ and $26 \mathrm{~S}$ proteasomes. Annu. Rev. Biochem. 65, 801-847.

8) Baumeister, W., Walz, J., Zuhl, F. and Seemuller, E. (1998) The proteasome: paradigm of a selfcompartmentalizing protease. Cell 92, 367-380.

9) Demartino, G. N. and Gillette, T. G. (2007) Proteasomes: machines for all reasons. Cell 129, 659-662.

10) Yoshimura, T., Kameyama, K., Takagi, T., Ikai, A., Tokunaga, F., Koide, T., Tanahashi, N., Tamura, T., Tanaka, K., Cejka, Z. et al. (1993) Molecular characterization of the '26S' proteasome complex from rat liver. J. Struct. Biol. 111, 200-211. 
11) Maupin-Furlow, J. A., Humbard, M. A., Kirkland, P. A., Li, W., Reuter, C. J., Wright, A. J. and Zhou, G. (2006) Proteasomes from structure to function: perspectives from Archaea. Curr. Top. Dev. Biol. 75, 125-169.

12) Groll, M., Ditzel, L., Lowe, J., Stock, D., Bochtler, M., Bartunik, H. D. and Huber, R. (1997) Structure of $20 \mathrm{~S}$ proteasome from yeast at 2.4 A resolution. Nature 386, 463-471.

13) Unno, M., Mizushima, T., Morimoto, Y., Tomisugi, Y., Tanaka, K., Yasuoka, N. and Tsukihara, T. (2002) The structure of the mammalian $20 \mathrm{~S}$ proteasome at $2.75 \mathrm{~A}$ resolution. Structure 10, 609-618.

14) Bochtler, M., Ditzel, L., Groll, M., Hartmann, C. and Huber, R. (1999) The proteasome. Annu. Rev. Biophys. Biomol. Struct. 28, 295-317.

15) Kloetzel, P. M. and Ossendorp, F. (2004) Proteasome and peptidase function in MHC-class-Imediated antigen presentation. Curr. Opin. Immunol. 16, 76-81.

16) Liu, C. W., Corboy, M. J., DeMartino, G. N. and Thomas, P. J. (2003) Endopoteolytic activity of the proteasome. Science 299, 408-411.

17) Jung, T. and Grune, T. (2008) The proteasome and its role in the degradation of oxidized proteins. IUBMB Life 60, 743-752.

18) Glickman, M. H., Rubin, D. M., Fried, V. A. and Finley, D. (1998) The regulatory particle of the Saccharomyces cerevisiae proteasome. Mol. Cell Biol. 18, 3149-3162.

19) Hanna, J. and Finley, D. (2007) A proteasome for all occasions. FEBS Lett. 581, 2854-2861.

20) Verma, R., Aravind, L., Oania, R., McDonald, W. H., Yates, J. R. 3rd, Koonin, E. V. and Deshaies, R. J. (2002) Role of Rpn11 metalloprotease in deubiquitination and degradation by the $26 \mathrm{~S}$ proteasome. Science 298, 611-615.

21) Hu, M., Li, P., Song, L., Jeffrey, P. D., Chenova, T. A., Wilkinson, K. D., Cohen, R. E. and Shi, Y. (2005) Structure and mechanisms of the proteasome-associated deubiquitinating enzyme USP14. EMBO J. 24, 3747-3756.

22) Hamazaki, J., Iemura, S., Natsume, T., Yashiroda, H., Tanaka, K. and Murata, S. (2006) A novel proteasome interacting protein recruits the deubiquitinating enzyme UCH37 to $26 \mathrm{~S}$ proteasomes. EMBO J. 25, 4524-4536.

23) Yao, T., Song, L., Xu, W., DeMartino, G. N., Florens, L., Swanson, S. K., Washburn, M. P., Conaway, R. C., Conaway, J. W. and Cohen, R. E. (2006) Proteasome recruitment and activation of the Uch37 deubiquitinating enzyme by Adrm1. Nat. Cell Biol. 8, 994-1002.

24) Hanna, J., Meides, A., Zhang, D. P. and Finley, D. (2007) A ubiquitin stress response induces altered proteasome composition. Cell 129, 747759 .

25) Rosenzweig, R., Osmulski, P. A., Gaczynska, M. and Glickman, M. H. (2008) The central unit within the $19 \mathrm{~S}$ regulatory particle of the proteasome. Nat. Struct. Mol. Biol. 15, 573-580.
26) Deveraux, Q., van Nocker, S., Mahaffey, D., Vierstra, R. and Rechsteiner, M. (1995) Inhibition of ubiquitin-mediated proteolysis by the Arabidopsis 26 S protease subunit S5a. J. Biol. Chem. 270, 29660-29663.

27) Schreiner, P., Chen, X., Husnjak, K., Randles, L., Zhang, N., Elsasser, S., Finley, D., Dikic, I., Walters, K. J. and Groll, M. (2008) Ubiquitin docking at the proteasome through a novel pleckstrin-homology domain interaction. Nature 453, 548-552.

28) Husnjak, K., Elsasser, S., Zhang, N., Chen, X., Randles, L., Shi, Y., Hofmann, K., Walters, K. J., Finley, D. and Dikic, I. (2008) Proteasome subunit Rpn13 is a novel ubiquitin receptor. Nature 453, 481-488.

29) Saeki, Y. and Tanaka, K. (2008) Cell biology: two hands for degradation. Nature 453, 460-461.

30) Lam, Y. A., Lawson, T. G., Velayutham, M., Zweier, J. L. and Pickart, C. M. (2002) A proteasomal ATPase subunit recognizes the polyubiquitin degradation signal. Nature 416, 763-767.

31) Kang, Y., Vossler, R. A., Diaz-Martinez, L. A., Winter, N. S., Clarke, D. J. and Walters, K. J. (2006) UBL/UBA ubiquitin receptor proteins bind a common tetraubiquitin chain. J. Mol. Biol. 356, 1027-1035.

32) Madura, K. (2004) Rad23 and Rpn10: perennial wallflowers join the melee. Trends Biochem. Sci. 29, 637-640.

33) Hartmann-Petersen, R. and Gordon, C. (2004) Integral UBL domain proteins: a family of proteasome interacting proteins. Semin. Cell Dev. Biol. 15, 247-259.

34) Moscat, J., Diaz-Meco, M. T. and Wooten, M. W. (2007) Signal integration and diversification through the p62 scaffold protein. Trends Biochem. Sci. 32, 95-100.

35) Elsasser, S. and Finley, D. (2005) Delivery of ubiquitinated substrates to protein-unfolding machines. Nat. Cell Biol. 7, 742-749.

36) Komatsu, M., Waguri, S., Koike, M., Sou, Y. S., Ueno, T., Hara, T., Mizushima, N., Iwata, J., Ezaki, J., Murata, S. et al. (2007) Homeostatic levels of p62 control cytoplasmic inclusion body formation in autophagy-deficient mice. Cell 131, 1149-1163.

37) Ichimura, Y., Kumanomidou, T., Sou, Y. S., Mizushima, T., Ezaki, J., Ueno, T., Kominami, E., Yamane, T., Tanaka, K. and Komatsu, M. (2008) Structural basis for sorting mechanism of p62 in selective autophagy. J. Biol. Chem. 283, $22847-22857$.

38) Elsasser, S., Gali, R. R., Schwickart, M., Larsen, C. N., Leggett, D. S., Muller, B., Feng, M. T., Tubing, F., Dittmar, G. A. and Finley, D. (2002) Proteasome subunit Rpn1 binds ubiquitin-like protein domains. Nat. Cell Biol. 4, 725730 .

39) Rechsteiner, M., Realini, C. and Ustrell, V. (2000) The proteasome activator 11 S REG (PA28) and 
class I antigen presentation. Biochem. J. 345, 115 .

40) Forster, A., Masters, E. I., Whitby, F. G. Robinson, H. and Hill, C. P. (2005) The 1.9 A structure of a proteasome-11S activator complex and implications for proteasome-PAN/PA700 interactions. Mol. Cell 18, 589-599.

41) Smith, D. M., Chang, S. C., Park, S., Finley, D., Cheng, Y. and Goldberg, A. L. (2007) Docking of the proteasomal ATPases' carboxyl termini in the $20 \mathrm{~S}$ proteasome's alpha ring opens the gate for substrate entry. Mol. Cell 27, 731-744.

42) Rabl, J., Smith, D. M., Yu, Y., Chang, S. C., Goldberg, A. L. and Cheng, Y. (2008) Mechanism of gate opening in the $20 \mathrm{~S}$ proteasome by the proteasomal ATPases. Mol. Cell 30, 360368.

43) Saeki, Y. and Tanaka, K. (2007) Unlocking the proteasome door. Mol. Cell 27, 865-867.

44) Liu, C. W., Li, X., Thompson, D., Wooding, K., Chang, T. L., Tang, Z., Yu, H., Thomas, P. J. and DeMartino, G. N. (2006) ATP binding and ATP hydrolysis play distinct roles in the function of 26S proteasome. Mol. Cell 24, 39-50.

45) Braun, B. C., Glickman, M., Kraft, R., Dahlmann, B., Kloetzel, P. M., Finley, D. and Schmidt, M. (1999) The base of the proteasome regulatory particle exhibits chaperone-like activity. Nat. Cell Biol. 1, 221-226.

46) Tanahashi, N., Yokota, K., Ahn, J. Y., Chung, C. H., Fujiwara, T., Takahashi, E., DeMartino, G. N., Slaughter, C. A., Toyonaga, T., Yamamura, K., Shimbara, N. and Tanaka, K. (1997) Molecular properties of the proteasome activator PA28 family proteins and gamma-interferon regulation. Genes Cells 2, 195211.

47) Wojcik, C., Tanaka, K., Paweletz, N., Naab, U. and Wilk, S. (1998) Proteasome activator (PA28) subunits, alpha, beta and gamma (Ki antigen) in NT2 neuronal precursor cells and HeLa S3 cells. Eur. J. Cell Biol. 77, 151-160.

48) Whitby, F. G., Masters, E. I., Kramer, L., Knowlton, J. R., Yao, Y., Wang, C. C. and Hill, C. P. (2000) Structural basis for the activation of $20 \mathrm{~S}$ proteasomes by $11 \mathrm{~S}$ regulators. Nature 408, 115-120.

49) Murata, S., Udono, H., Tanahashi, N., Hamada, N., Watanabe, K., Adachi, K., Yamano, T., Yui, K., Kobayashi, N., Kasahara, M. et al. (2001) Immunoproteasome assembly and antigen presentation in mice lacking both PA28alpha and PA28beta. EMBO J. 20, 5898-5907.

50) Tanaka, K. and Kasahara, M. (1998) The MHC class I ligand-generating system: roles of immunoproteasomes and the interferon-gamma-inducible proteasome activator PA28. Immunol. Rev. 163, 161-176.

51) Kloetzel, P. M. (2001) Antigen processing by the proteasome. Nat. Rev. Mol. Cell Biol. 2, 179187.

52) Rock, K. L., York, I. A., Saric, T. and Goldberg,
A. L. (2002) Protein degradation and the generation of MHC class I-presented peptides. Adv. Immunol. 80, 1-70.

53) Murata, S., Kawahara, H., Tohma, S., Yamamoto, K., Kasahara, M., Nabeshima, Y., Tanaka, K. and Chiba, T. (1999) Growth retardation in mice lacking the proteasome activator PA28gamma. J. Biol. Chem. 274, 38211-38215.

54) Zhang, Z. and Zhang, R. (2008) Proteasome activator PA28 gamma regulates p53 by enhancing its MDM2-mediated degradation. EMBO J. 27, 852-864.

55) Li, X., Lonard, D. M., Jung, S. Y., Malovannaya, A., Feng, Q., Qin, J., Tsai, S. Y., Tsai, M. J. and O'Malley, B. W. (2006) The SRC-3/AIB1 coactivator is degraded in a ubiquitin- and ATPindependent manner by the REGgamma proteasome. Cell 124, 381-392.

56) Li, X., Amazit, L., Long, W., Lonard, D. M., Monaco, J. J. and O'Malley, B. W. (2007) Ubiquitin- and ATP-independent proteolytic turnover of p21 by the REGgamma-proteasome pathway. Mol. Cell 26, 831-842.

57) Chen, X., Barton, L. F., Chi, Y., Clurman, B. E. and Roberts, J. M. (2007) Ubiquitin-independent degradation of cell-cycle inhibitors by the REGgamma proteasome. Mol. Cell 26, 843-852.

58) Zannini, L., Lecis, D., Buscemi, G., Carlessi, L., Gasparini, P., Fontanella, E., Lisanti, S., Barton, L. and Delia, D. (2008) REGgamma proteasome activator is involved in the maintenance of chromosomal stability. Cell Cycle 7, $504-512$.

59) Mao, I., Liu, J., Li, X. and Luo, H. (2008) REGgamma, a proteasome activator and beyond. Cell Mol. Life Sci. (in press).

60) Moriishi, K., Mochizuki, R., Moriya, K., Miyamoto, H., Mori, Y., Abe, T., Murata, S., Tanaka, K., Miyamura, T., Suzuki, T. et al. (2007) Critical role of PA28gamma in hepatitis $\mathrm{C}$ virus-associated steatogenesis and hepatocarcinogenesis. Proc. Natl. Acad. Sci. USA 104, $1661-1666$.

61) Tanahashi, N., Murakami, Y., Minami, Y., Shimbara, N., Hendil, K. B. and Tanaka, K. (2000) Hybrid proteasomes. Induction by interferon-gamma and contribution to ATP-dependent proteolysis. J. Biol. Chem. 275, 1433614345.

62) Cascio, P., Call, M., Petre, B. M., Walz, T. and Goldberg, A. L. (2002) Properties of the hybrid form of the $26 \mathrm{~S}$ proteasome containing both $19 \mathrm{~S}$ and PA28 complexes. EMBO J. 21, 2636-2645.

63) Kopp, F., Dahlmann, B. and Kuehn, L. (2001) Reconstitution of hybrid proteasomes from purified PA700-20 S complexes and PA28alphabeta activator: ultrastructure and peptidase activities. J. Mol. Biol. 313, 465-471.

64) Murakami, Y., Matsufuji, S., Kameji, T., Hayashi, S., Igarashi, K., Tamura, T., Tanaka, K. and Ichihara, A. (1992) Ornithine decarboxylase is 
degraded by the $26 \mathrm{~S}$ proteasome without ubiquitination. Nature 360, 597-599.

65) Ustrell, V., Hoffman, L., Pratt, G. and Rechsteiner, M. (2002) PA200, a nuclear proteasome activator involved in DNA repair. EMBO J. 21, 3516-3525.

66) Schmidt, M., Haas, W., Crosas, B., Santamaria, P. G., Gygi, S. P., Walz, T. and Finley, D. (2005) The HEAT repeat protein Blm10 regulates the yeast proteasome by capping the core particle. Nat. Struct. Mol. Biol. 12, 294-303.

67) Fehlker, M., Wendler, P., Lehmann, A. and Enenkel, C. (2003) Blm3 is part of nascent proteasomes and is involved in a late stage of nuclear proteasome assembly. EMBO Rep. 4, 959-963.

68) Marques, A. J., Glanemann, C., Ramos, P. C. and Dohmen, R. J. (2007) The C-terminal extension of the beta7 subunit and activator complexes stabilize nascent $20 \mathrm{~S}$ proteasomes and promote their maturation. J. Biol. Chem. 282, 3486934876 .

69) Ortega, J., Heymann, J. B., Kajava, A. V., Ustrell, V., Rechsteiner, M. and Steven, A. C. (2005) The axial channel of the 20S proteasome opens upon binding of the PA200 activator. J. Mol. Biol. 346, 1221-1227.

70) Iwanczyk, J., Sadre-Bazzaz, K., Ferrell, K., Kondrashkina, E., Formosa, T., Hill, C. P. and Ortega, J. (2006) Structure of the Blm10-20 S proteasome complex by cryo-electron microscopy. Insights into the mechanism of activation of mature yeast proteasomes. J. Mol. Biol. 363, 648-659.

71) Lehmann, A., Jechow, K. and Enenkel, C. (2008) Blm10 binds to pre-activated proteasome core particles with open gate conformation. EMBO Rep. (in press).

72) Blickwedehl, J., Agarwal, M., Seong, C., Pandita, R. K., Melendy, T., Sung, P., Pandita, T. K. and Bangia, N. (2008) Role for proteasome activator PA200 and postglutamyl proteasome activity in genomic stability. Proc. Natl. Acad. Sci. USA 105, 16165-16170.

73) Khor, B., Bredemeyer, A. L., Huang, C. Y., Turnbull, I. R., Evans, R., Maggi, L. B. Jr., White, J. M., Walker, L. M., Carnes, K., Hess, R. A. and Sleckman, B. P. (2006) Proteasome activator PA200 is required for normal spermatogenesis. Mol. Cell Biol. 26, 2999-3007.

74) McCutchen-Maloney, S. L., Matsuda, K., Shimbara, N., Binns, D. D., Tanaka, K. Slaughter, C. A. and DeMartino, G. N. (2000) cDNA cloning, expression, and functional characterization of PI31, a proline-rich inhibitor of the proteasome. J. Biol. Chem. 275, 1855718565

75) Zaiss, D. M., Standera, S., Kloetzel, P. M. and Sijts, A. J. (2002) PI31 is a modulator of proteasome formation and antigen processing. Proc. Natl. Acad. Sci. USA 99, 14344-14349.

76) Kirk, R., Laman, H., Knowles, P. P., Murray-
Rust, J., Lomonosov, M., Mezianeel, K. and McDonald, N. Q. (2008) Structure of a conserved dimerization domain within the F-box protein Fbxo7 and the PI31 proteasome inhibitor. J. Biol. Chem. 283, 22325-22335.

77) Rechsteiner, M. and Hill, C. P. (2005) Bilizing the proteolytic machine: cell biological roles of proteasome activators and inhibitors. Trends Cell Biol. 15, 27-33.

78) Anbanandam, A., Albarado, D. C., Tirziu, D. C., Simons, M. and Veeraraghavan, S. (2008) Molecular basis for proline- and arginine-rich peptide inhibition of proteasome. J. Mol. Biol. 384, 219-227.

79) Smalle, J. and Vierstra, R. D. (2004) The ubiquitin $26 \mathrm{~S}$ proteasome proteolytic pathway. Annu. Rev. Plant Biol. 55, 555-590.

80) Belote, J. M. and Zhong, L. (2005) Proteasome gene duplications in mammals, flies and plants. Recent Res. Devel. Gene \& Genomes 1, 107-129.

81) Fu, H., Doelling, J. H., Arendt, C. S., Hochstrasser, M. and Vierstra, R. D. (1998) Molecular organization of the $20 \mathrm{~S}$ proteasome gene family from Arabidopsis thaliana. Genetics 149, 677-692.

82) Fehling, H. J., Swat, W., Laplace, C., Kuhn, R., Rajewsky, K., Muller, U. and von Boehmer, H. (1994) MHC class I expression in mice lacking the proteasome subunit LMP-7. Science 265, $1234-1237$

83) van Kaer, L., Ashton-Rickardt, P. G., Eichelberger, M., Gaczynska, M., Nagashima, K., Rock, K. L., Goldberg, A. L., Doherty, P. C. and Tonegawa, S. (1994) Altered peptidase and viral-specific $\mathrm{T}$ cell response in LMP2 mutant mice. Immunity 1, 533-541.

84) Barton, L. F., Runnels, H. A., Schell, T. D., Cho, Y., Gibbons, R., Tevethia, S. S., Deepe, G. S. Jr. and Monaco, J. J. (2004) Immune defects in 28$\mathrm{kDa}$ proteasome activator gamma-deficient mice. J. Immunol. 172, 3948-3954.

85) Kasahara, M., Hayashi, M., Tanaka, K., Inoko, H., Sugaya, K., Ikemura, T. and Ishibashi, T. (1996) Chromosomal localization of the proteasome $\mathrm{Z}$ subunit gene reveals an ancient chromosomal duplication involving the major histocompatibility complex. Proc. Natl. Acad. Sci. USA 93, 9096-9101.

86) Murata, S., Sasaki, K., Kishimoto, T., Niwa, S., Hayashi, H., Takahama, Y. and Tanaka, K. (2007) Regulation of $\mathrm{CD}^{+} \mathrm{T}$ cell development by thymus-specific proteasomes. Science $\mathbf{3 1 6}$, 1349-1353.

87) Murata, S., Takahama, Y. and Tanaka, K. (2008) Thymoproteasome: probable role in generating positively selecting peptides. Curr. Opin. Immunol. 20, 192-196.

88) Takahama, Y., Tanaka, K. and Murata, S. (2008) Modest cortex and promiscuous medulla for thymic repertoire formation. Trends Immunol. 29, 251-255.

89) Nitta, T., Murata, S., Ueno, T., Tanaka, K. and 
Takahama, Y. (2008) Thymic environments of T-cell repertoire formation. Adv. Immunol. (in press).

90) Zhong, L. and Belote, J. M. (2007) The testisspecific proteasome subunit Prosalpha6 T of D. melanogaster is required for individualization and nuclear maturation during spermatogenesis. Development 134, 3517-3525.

91) Kawahara, H., Kasahara, M., Nishiyama, A., Ohsumi, K., Goto, T., Kishimoto, T., Saeki, Y., Yokosawa, H., Shimbara, N., Murata, S. et al. (2000) Developmentally regulated, alternative splicing of the Rpn10 gene generates multiple forms of $26 \mathrm{~S}$ proteasomes. EMBO J. 19, 4144-4153.

92) Hamazaki, J., Sasaki, K., Kawahara, H., Hisanaga, S., Tanaka, K. and Murata, S. (2007) Rpn10-mediated degradation of ubiquitinated proteins is essential for mouse development. Mol. Cell Biol. 27, 6629-6638.

93) Stanhill, A., Haynes, C. M., Zhang, Y., Min, G., Steele, M. C., Kalinina, J., Martinez, E., Pickart, C. M., Kong, X. P. and Ron, D. (2006) An arsenite-inducible 19S regulatory particle-associated protein adapts proteasomes to proteotoxicity. Mol. Cell 23, 875-885.

94) Yun, C., Stanhill, A., Yang, Y., Zhang, Y., Haynes, C. M., Xu, C. F., Neubert, T. A. Mor, A., Philips, M. R. and Ron, D. (2008) Proteasomal adaptation to environmental stress links resistance to proteotoxicity with longevity in Caenorhabditis elegans. Proc. Natl. Acad. Sci. USA 105, 7094-7099.

95) Ellis, R. J. (2006) Molecular chaperones: assisting assembly in addition to folding. Trends Biochem. Sci. 31, 395-401.

96) Kusmierczyk, A. R. and Hochstrasser, M. (2008) Some assembly required: dedicated chaperones in eukaryotic proteasome biogenesis. Biol. Chem. 389, 1143-1151.

97) Ramos, P. C. and Dohmen, R. J. (2008) PACemakers of proteasome core particle assembly. Structure 16, 1296-304.

98) Murata, S., Yashiroda, H. and Tanaka, K. (2009) Molecular mechanisms of proteasome assembly. Nat. Rev. Mol. Cell Biol. (in press).

99) Rosenzweig, R. and Glickman, M. H. (2008) Chaperone-driven proteasome assembly. Biochem. Soc. Trans. 36, 807-812.

100) Hirano, Y., Hendil, K. B., Yashiroda, H., Iemura, S., Nagane, R., Hioki, Y., Natsume, T., Tanaka, K. and Murata, S. (2005) A heterodimeric complex that promotes the assembly of mammalian 20S proteasomes. Nature 437, 13811385.

101) Li, X., Kusmierczyk, A. R., Wong, P., Emili, A. and Hochstrasser, M. (2007) Beta-Subunit appendages promote $20 \mathrm{~S}$ proteasome assembly by overcoming an Ump1-dependent checkpoint. EMBO J. 26, 2339-2349.

102) Hirano, Y., Hayashi, H., Iemura, S., Hendil, K. B., Niwa, S., Kishimoto, T., Kasahara, M.,
Natsume, T., Tanaka, K. and Murata, S. (2006) Cooperation of multiple chaperones required for the assembly of mammalian 20S proteasomes. Mol. Cell 24, 977-984.

103) Le Tallec, B., Barrault, M. B., Courbeyrette, R., Guerois, R., Marsolier-Kergoat, M. C. and Peyroche, A. (2007) 20S proteasome assembly is orchestrated by two distinct pairs of chaperones in yeast and in mammals. Mol. Cell 27, 660-674.

104) Yashiroda, H., Mizushima, T., Okamoto, K., Kameyama, T., Hayashi, H., Kishimoto, T., Niwa, S., Kasahara, M., Kurimoto, E., Sakata, E. et al. (2008) Crystal structure of a chaperone complex that contributes to the assembly of yeast $20 \mathrm{~S}$ proteasomes. Nat. Struct. Mol. Biol. 15, 228-236.

105) Kusmierczyk, A. R., Kunjappu, M. J., Funakoshi, M. and Hochstrasser, M. (2008) A multimeric assembly factor controls the formation of alternative $20 \mathrm{~S}$ proteasomes. Nat. Struct. Mol. Biol. 15, 237-244.

106) Hoyt, M. A., McDonough, S., Pimpl, S. A., Scheel, H., Hofmann, K. and Coffino, P. (2008) A genetic screen for Saccharomyces cerevisiae mutants affecting proteasome function, using a ubiquitin-independent substrate. Yeast 25, 199217.

107) Shinde, U. and Inouye, M. (2000) Intramolecular chaperones: polypeptide extensions that modulate protein folding. Semin Cell Dev. Biol. 11, $35-44$.

108) De, M., Jayarapu, K., Elenich, L., Monaco, J. J., Colbert, R. A. and Griffin, T. A. (2003) Beta 2 subunit propeptides influence cooperative proteasome assembly. J. Biol. Chem. 278, 61536159 .

109) Chen, P. and Hochstrasser, M. (1996) Autocatalytic subunit processing couples active site formation in the $20 \mathrm{~S}$ proteasome to completion of assembly. Cell 86, 961-972.

110) Hirano, Y., Kaneko, T., Okamoto, K., Bai, M., Yashiroda, H., Furuyama, K., Kato, K., Tanaka, K. and Murata, S. (2008) Dissecting beta-ring assembly pathway of the mammalian 20S proteasome. EMBO J. 27, 2204-2213.

111) Ramos, P. C., Hockendorff, J., Johnson, E. S., Varshavsky, A. and Dohmen, R. J. (1998) Ump1p is required for proper maturation of the 20S proteasome and becomes its substrate upon completion of the assembly. Cell 92, 489499.

112) Witt, E., Zantopf, D., Schmidt, M., Kraft, R., Kloetzel, P. M. and Kruger, E. (2000) Characterisation of the newly identified human Ump1 homologue POMP and analysis of LMP7(beta 5i) incorporation into $20 \mathrm{~S}$ proteasomes. J. Mol. Biol. 301, 1-9.

113) Griffin, T. A., Slack, J. P., McCluskey, T. S., Monaco, J. J. and Colbert, R. A. (2000) Identification of proteassemblin, a mammalian homologue of the yeast protein, Ump1p, that is 
required for normal proteasome assembly. Mol. Cell Biol. Res. Commun. 3, 212-217.

114) Griffin, T. A., Nandi, D., Cruz, M., Fehling, H. J., Kaer, L. V., Monaco, J. J. and Colbert, R. A. (1998) Immunoproteasome assembly: cooperative incorporation of interferon gamma (IFNgamma)-inducible subunits. J. Exp. Med. 187, 97-104.

115) Kingsbury, D. J., Griffin, T. A. and Colbert, R. A. (2000) Novel propeptide function in $20 \mathrm{~S}$ proteasome assembly influences beta subunit composition. J. Biol. Chem. 275, 24156-24162.

116) Heink, S., Ludwig, D., Kloetzel, P. M. and Kruger, E. (2005) IFN-gamma-induced immune adaptation of the proteasome system is an accelerated and transient response. Proc. Natl. Acad. Sci. USA 102, 9241-9246.

117) Isono, E., Nishihara, K., Saeki, Y., Yashiroda, H., Kamata, N., Ge, L., Ueda, T., Kikuchi, Y., Tanaka, K., Nakano, A. and Toh-e, A. (2007) The assembly pathway of the $19 \mathrm{~S}$ regulatory particle of the yeast $26 \mathrm{~S}$ proteasome. Mol. Biol. Cell 18, 569-580.

118) Isono, E., Saito, N., Kamata, N., Saeki, Y. and Toh, E. A. (2005) Functional analysis of Rpn6p, a lid component of the $26 \mathrm{~S}$ proteasome, using temperature-sensitive rpn6 mutants of the yeast Saccharomyces cerevisiae. J. Biol. Chem. 280, $6537-6547$

119) Imai, J., Maruya, M., Yashiroda, H., Yahara, I. and Tanaka, K. (2003) The molecular chaperone Hsp90 plays a role in the assembly and maintenance of the $26 \mathrm{~S}$ proteasome. EMBO J. 22, $3557-3567$.

120) Kleijnen, M. F., Roelofs, J., Park, S., Hathaway, N. A., Glickman, M., King, R. W. and Finley, D. (2007) Stability of the proteasome can be regulated allosterically through engagement of its proteolytic active sites. Nat. Struct. Mol. Biol. 14, 1180-1188.

121) Babbitt, S. E., Kiss, A., Deffenbaugh, A. E., Chang, Y. H., Bailly, E., Erdjument-Bromage, H., Tempst, P., Buranda, T., Sklar, L. A., Baumler, J. et al. (2005) ATP hydrolysisdependent disassembly of the $26 \mathrm{~S}$ proteasome is part of the catalytic cycle. Cell 121, 553-565.

122) Kriegenburg, F., Seeger, M., Saeki, Y., Tanaka, K., Lauridsen, A. M. B., Hartmann-Petersen, R. and Hendil, K. B. (2008) Mammalian 26S proteasomes remain intact during protein degradation. Cell 135, 355-365.

123) Verma, R., Chen, S., Feldman, R., Schieltz, D., Yates, J., Dohmen, J. and Deshaies, R. J. (2000) Proteasomal proteomics: identification of nucleotide-sensitive proteasome-interacting proteins by mass spectrometric analysis of affinitypurified proteasomes. Mol. Biol. Cell 11, 34253439 .

124) Guerrero, C., Tagwerker, C., Kaiser, P. and Huang, L. (2006) An integrated mass spectrometry-based proteomic approach: quantitative analysis of tandem affinity-purified in vivo cross-linked protein complexes (QTAX) to decipher the $26 \mathrm{~S}$ proteasome-interacting network. Mol. Cell Proteomics 5, 366-378.

125) Wang, X., Chen, C. F., Baker, P. R., Chen, P. L., Kaiser, P. and Huang, L. (2007) Mass spectrometric characterization of the affinity-purified human $26 \mathrm{~S}$ proteasome complex. Biochemistry 46, 3553-65.

126) Leggett, D. S., Hanna, J., Borodovsky, A., Crosas, B., Schmidt, M., Baker, R. T., Walz, T., Ploegh, H. and Finley, D. (2002) Multiple associated proteins regulate proteasome structure and function. Mol. Cell 10, 495-507.

127) Gorbea, C., Goellner, G. M., Teter, K., Holmes, R. K. and Rechsteiner, M. (2004) Characterization of mammalian Ecm29, a $26 \mathrm{~S}$ proteasome-associated protein that localizes to the nucleus and membrane vesicles. J. Biol. Chem. 279, 54849-54861.

128) Tonoki, A., Kuranaga, E., Tomioka, T., Hamazaki, J., Murata, S., Tanaka, K. and Miura, M. (2009) Genetic evidence linking agedependent attenuation of the $26 \mathrm{~S}$ proteasome with aging process. Mol. Cell Biol. (in press).

129) Tanaka, K., Yoshimura, T., Tamura, T., Fujiwara, T., Kumatori, A. and Ichihara, A. (1990) Possible mechanism of nuclear translocation of proteasomes. FEBS Lett. 271, 4146.

130) Ferdous, A., Kodadek, T. and Johnston, S. A. (2002) A nonproteolytic function of the 19S regulatory subunit of the $26 \mathrm{~S}$ proteasome is required for efficient activated transcription by human RNA polymerase II. Biochemistry 41, 12798-12805.

131) Collins, G. A. and Tansey, W. P. (2006) The proteasome: a utility tool for transcription? Curr. Opin. Genet. Dev. 16, 197-202.

132) Russell, S. J., Reed, S. H., Huang, W., Friedberg, E. C. and Johnston, S. A. (1999) The 19S regulatory complex of the proteasome functions independently of proteolysis in nucleotide excision repair. Mol. Cell 3, 687-695.

133) Reed, S. H. and Gillette, T. G. (2007) Nucleotide excision repair and the ubiquitin proteasome pathway-do all roads lead to Rome? DNA Repair (Amst.) 6, 149-156.

134) Wahlman, J., DeMartino, G. N., Skach, W. R., Bulleid, N. J., Brodsky, J. L. and Johnson, A. E. (2007) Real-time fluorescence detection of ERAD substrate retrotranslocation in a mammalian in vitro system. Cell 129, 943-955.

135) Tanaka, K. (1998) Proteasomes: structure and biology. J. Biochem. (Tokyo) 123, 195-204.

136) Goldberg, A. L. (2007) Functions of the proteasome: from protein degradation and immune surveillance to cancer therapy. Biochem. Soc. Trans. 35, 12-17.

137) Jensen, T. J., Loo, M. A., Pind, S., Williams, D. B., Goldberg, A. L. and Riordan, J. R. (1995) Multiple proteolytic systems, including the proteasome, contribute to CFTR processing. Cell 
83, 129-135.

138) Meng, L., Mohan, R., Kwok, B. H., Elofsson, M., Sin, N. and Crews, C. M. (1999) Epoxomicin, a potent and selective proteasome inhibitor, exhibits in vivo antiinflammatory activity. Proc. Natl. Acad. Sci. USA 96, 10403-10408.

139) Adams, J. (2004) The proteasome: a suitable antineoplastic target. Nat. Rev. Cancer 4, 349360.

140) Voorhees, P. M. and Orlowski, R. Z. (2006) The proteasome and proteasome inhibitors in cancer therapy. Annu. Rev. Pharmacol. Toxicol. 46, 189-213.
141) Prudhomme, J., McDaniel, E., Ponts, N., Bertani, S., Fenical, W., Jensen, P. and Le Rock, K. (2008) Marine actinomycetes: a new source of compounds against the human malaria parasite. PloS One 3, e2335.

142) Yang, H., Landis-Piwowar, K. R., Chen, D., Milacic, V. and Dou, Q. P. (2008) Natural compounds with proteasome inhibitory activity for cancer prevention and treatment. Curr. Protein Pept. Sci. 9, 227-239.

(Received Sept. 19, 2008; accepted Nov. 28, 2008)

\section{Profile}

Keiji Tanaka was born in 1949 and started his research career in 1972 with studies on the amino acid and protein metabolism in the Institute of Enzyme Research, after graduating from the Faculty of Medicine (School of Nutrition) at The University of Tokushima. He received his Ph.D. from The University of Tokushima in 1980, working on the hepatic protein metabolism. He was promoted to assistant professor in 1976 and associate professor in 1995 at the Institute for Enzyme Research at The University of Tokushima, and head of the Department of Molecular Oncology in 1996 and Vice-Director in 2002 at The Tokyo Metropolitan Institute of Medical Science. He is an acting director at The Tokyo Metropolitan Institute of Medical Science since 2006. Over the past 25 years, he focused on elucidating the structure and

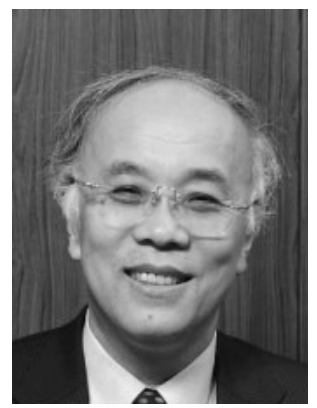
molecular/physiological functions of the proteasome. The discoveries of proteasomes in 1988, immunoproteasomes in 1994, hybrid proteasomes in 2000, and thymoproteasomes in 2007 are the highlights of his study. His current research interests include intracellular proteolysis mediated by the proteasome, ubiquitin, and autophagy system in eukaryotes in general. He was awarded the Naito Memorial Foundation Prize in 2003, the Asahi Culture Prize and the Uehara Prize in 2004, and the Toray Science Technology Prize in 2007. At present he is a gust professor of Ochanomizu Woman's University, Tokyo Medical and Dental University, The University of Tokyo Graduate School of Frontier Sciences, Juntendo University School of Medicine, and Niigata University School of Medicine. 